\title{
Experimental stress in inflammatory rheumatic diseases: a review of psychophysiological stress responses
}

\author{
Sabine JM de Brouwer*1, Floris W Kraaimaat ${ }^{1}$, Fred CGJ Sweep², Marjonne CW Creemers 3 , Timothy RDJ Radstake3, \\ Antoinette IM van Laarhoven ${ }^{1}$, Piet LCM van Riel ${ }^{3}$ and Andrea WM Evers'
}

\begin{abstract}
Introduction: Stressful events are thought to contribute to the aetiology, maintenance and exacerbation of rheumatic diseases. Given the growing interest in acute stress responses and disease, this review investigates the impact of reallife experimental psychosocial, cognitive, exercise and sensory stressors on autonomic, neuroendocrine and immune function in patients with inflammatory rheumatic diseases.

Methods: Databases Medline, PsychINFO, Embase, Cinahl and Pubmed were screened for studies (1985 to 2009) investigating physiological stress responses in inflammatory rheumatic diseases. Eighteen articles met the inclusion criteria.

Results: Results suggest that immune function may be altered in response to a stressor; such alterations could contribute to the maintenance or exacerbation of inflammatory rheumatic diseases during stressful events in daily life.

Conclusions: This review emphasizes the need for more experimental research in rheumatic populations with controlled stress paradigms that include a follow-up with multiple evaluation points, simultaneous assessment of different physiological stress systems, and studying factors contributing to specific physiological responses, such as stress appraisal.
\end{abstract}

\section{Introduction}

Stress is widely recognized as an important risk factor in the aetiology of inflammatory rheumatic diseases [1-5]. An adaptational stress response involves the activation of both the hypothalamus-pituitary-adrenal axis (HPA axis) [6] and the autonomic nervous system (ANS) [7], and both stress axes are thought to communicate bidirectionally with the immune system [7-10]. Because many rheumatic diseases are characterized by immune-mediated joint inflammation, stressful events might contribute to the aetiology, maintenance and exacerbation of rheumatic diseases $[11,12]$. Recent advances in psychoneuroimmunology have provided insight into the complex mechanisms by which stressors might acutely affect the

* Correspondence: S.deBrouwer@mps.umcn.nl

1 Department of Medical Psychology, Radboud University Nijmegen Medical Centre, P.O. Box 9101, 6500 HB Nijmegen, The Netherlands

Full list of author information is available at the end of the article

See related editorial by Hassett and Clauw, http://arthritis-research.com/content/12/ $3 / 123$

(C) 2010 de Brouwer et al.; licensee BioMed Central Ltd. This is an open access article distributed under the terms of the Creative Commons Attribution License (http://creativecommons.org/licenses/by/2.0), which permits unrestricted use, distribution, and reproduction in any medium, provided the original work is properly cited. body's immune system [13-16]. However, little attention has been paid to whether and how different short-term experimental stressors influence the separate pathways of the physiological stress response system (ANS, HPA axis, immune system) in patients with inflammatory rheumatic diseases.

Perception of an external stressful stimulus prompts the activation of various physiological systems that together define the body's stress response, which is aimed at re-establishing homeostasis. The physiological stress response is mainly coordinated by the hypothalamus, with activation of the ANS and the pituitary and adrenal glands (HPA axis) resulting in the release of catecholamines and cortisol, respectively $[1,9,17]$. These stress hormones, supposedly acting via $\beta$ - and $\alpha$-adrenergic as well as glucocorticoid receptors, down-regulate immune and inflammatory processes; however, these processes also influence the central nervous system (CNS) [7,18-20]. Circulating cytokines (for example, 
tumor necrosis factor $\alpha$ (TNF- $\alpha$ ), interleukin (IL)- 6 and IL-1) and activated immune cells, markers of inflammation, activate both (intermediates of) the HPA axis and the ANS. Chronically elevated levels of cytokines, as occur during long-term inflammation, might lead to changes in HPA axis and ANS activity [21]. Moreover, the bidirectional relationship between the CNS and immune system implies that the physiological response to real-life stressors could contribute to the pathophysiology of inflammatory diseases [1-5]. How these three systems, the ANS, the HPA axis and the immune system, act in response to a stressful event in rheumatic disorders is not well understood.

Although the laboratory setting is not a natural environment, it allows control of key factors in the delivery of stress and observation of its effects and reduces many sources of bias and individual differences [16,22]. The literature on acute psychoneuroimmunological and psychoendocrinological responses to experimental stress in healthy individuals is still increasing. Studies of healthy populations suggest that experimental psychological and physical stressors not only activate the ANS [23] and the HPA axis [24], but also influence the immune system by activating innate immunity, as reflected by increased numbers of natural killer $(\mathrm{NK})$ cells and the production of pro-inflammatory IL-6 $[15,16]$. Moreover, these different physiological systems (ANS, HPA axis and immune system) seem to work in an interdependent fashion [25].

Despite the possible detrimental physiological effects of stress in patients with inflammatory rheumatic diseases, such as an altered disease course, little is known about acute-phase reactants of experimentally induced stress (both autonomic, neuroendocrine and immune). Reviews of acute physiological stress responses have either focussed on one [16,24] or two [2] stress response systems only (for example, ANS and/or neuroendocrine system), and included either only patients with rheumatoid arthritis [2] or a heterogeneous group of both healthy participants and various patient populations [16]. In addition, studies of the relationship between stress and inflammatory rheumatic diseases have often used experimental stressors that do not necessarily mimic real-life stressors. Different types of time-limited experimental stressors have been identified, namely, physical stressors (autonomic function tests, exercise), physiological stressors (corticotropin-releasing hormone $(\mathrm{CRH})$ and (nor)epinephrine infusions, insulin tolerance test and dexamethasone suppression test) and psychological stressors (cognitive tests, public speaking) [2]. Many studies have investigated the effects of these types of stress on components of the stress response system, such as the ANS or the HPA axis, but external validity of these studies of stress is questionable. The prevalence of cardiovascular dysfunction is high after standard tests of autonomic function [26], such as the Valsalva manoeuvre, deep breathing, orthostatic tests, and sustained handgrip. While these tests may trigger autonomic responses, it is not known whether they activate the stress response system and alter neuroendocrine or immune function. HPA axis function has been investigated extensively by challenging specific parts of the HPA axis by means of infusion of CRH, synthetic glucocorticoids, or cytokines $[27,28]$. Although alterations in HPA axis responsiveness at a hypothalamic, pituitary or adrenal level have been reported, more subtle changes in HPA functioning have also been suggested to occur $[27,28]$. While injection studies might shed some light on possible altered neuroendocrine responses, the anti-inflammatory effects of exogenously administered glucocorticoids are not necessarily mirrored by increased secretion of endogenous glucocorticoids in response to a real-life stressor. Thus the question remains to what extent different types of experimental stressors that mimic real-life stressful events (for example, psychological stressors and physical exercise) are able to induce an autonomic, neuroendocrine and immune response in patients with inflammatory rheumatic diseases.

To the best of our knowledge this is the first review to investigate whether and how different experimental stressors mimicking real-life stressful events (psychosocial, cognitive, exercise and sensory stressors) influence physiological responses at the three levels (ANS, HPA-axis, immune system) in patients with prototypic inflammatory rheumatic diseases (for example, rheumatoid arthritis (RA) and systemic lupus erythematosus (SLE).

\section{Materials and methods}

This review is limited to studies involving patients with inflammatory rheumatic diseases who were exposed to a time-limited experimental stressor to assess the autonomic, neuroendocrine, and/or immune responses to stress.

\section{Search strategy}

To identify studies, the electronic bibliographic databases MEDLINE, PsychINFO, Embase, Cinahl and Pubmed databases were searched, using the key words rheum*, (idiopathic or psoriatic) arthr", spondylitis, sclerosis and lupus in combination with the terms stress and either cortisol or immun* or epinephrine or endocrin* or autonom* or hypothalam" or HPA. In addition, reference sections of the articles and review papers were hand-searched for relevant articles on psychological and physical stressors and inflammatory rheumatic diseases.

Inclusion criteria were studies published after 1985 in English peer-reviewed journals; evaluation of an experimental laboratory stress task that induces psychological and/or physical (exercise) stress and/or sensory stress (for 
example, thermoceptive (cold/heat), visual (light), auditive (noise)) by means of a time-limited experimental stressor; patients diagnosed with systemic inflammatory rheumatic diseases, such as RA, juvenile idiopathic arthritis (JIA), ankylosing spondylitis, systemic sclerosis, or SLE; control group consisting of either healthy participants or participants without a systemic inflammatory rheumatic disease, such as osteoarthritis; use of (neuro)endocrine variables (for example, cortisol levels), ANS variables (for example, heart rate, skin conductance, (nor)epinephrine levels), or immune variables (for example, number of leucocytes or lymphocytes, subsets of lymphocytes, interleukin levels) as outcomes. Exclusion criteria were pharmacological studies involving $\mathrm{CRH}$, glucocorticoids, insulin or (nor)epinephrine infusions; studies evaluating a battery of standard autonomic function tests, which include deep breathing, the Valsalva manoeuvre, posture changes, and sustained handgrip, unless they were part of a paradigm with a psychological and/or exercise and/or sensory stressor [29-33]; assessment of anaerobic threshold, peak oxygen consumption, lactate threshold [34,35], fibrinogen and prothrombin time [36]. If a research group published more than one article on the same experimental study but evaluated different outcome measures, both articles were included in the review $[29,30,36,37]$.

Conclusions were based on (uni- or multivariate) statistics for within-group and/or between-groups differences. If studies reported significant between-group differences in repeated measures ANOVAs, baseline values between groups were assumed to be different, unless stated otherwise. If studies did not report significant (within- or between-) group differences, it was assumed that none were found. If studies did not provide statistical analyses, findings were based on mean ( \pm standard error of the mean (SEM) or standard deviation (SD)) or median values. If those values were ambiguous, no conclusions were drawn.

\section{Results \\ Participants \\ Patient groups}

Sixteen studies (18 articles) met the inclusion criteria. The study sample characteristics are summarized in Table 1. Nine studies included patients with RA [29,30,34-42] and seven patients with SLE [31,33,35,40,43-45]. Two of these studies involved both types of patients $[35,40]$. In addition, one study included patients with JIA [46] and one study included a heterogeneous group of patients with inflammatory arthritis (RA, psoriatic arthritis, ankylosing spondylitis and fibrositis) [32]. Fifteen studies included healthy participants without systemic inflammatory rheumatic disease as control and one study, patients with osteoarthritis [36,37]. In three studies a second control group was added, consisting of either patients with myofascial pain [32], patients with sarcoidosis [43], or participants taking corticosteroids [44]. Most studies have relatively small sample sizes ( $\mathrm{N}=10$ to 20 per group), with even smaller sample sizes $(\mathrm{N}<10)$ in two studies [35,40]. In addition, patient samples are probably heterogeneous because, for example, strict exclusion criteria regarding comorbidities are lacking in half of all studies [32,33,35,38,42-44,46]. With regard to diagnostic criteria, one study even included also patients with non-inflammatory arthritis [32]. There is also a high variability in pharmacotherapy profiles, but overall these profiles were well-defined for the various classes of drugs (see Table 1), except in two studies $[32,33]$. To control for the effects of pharmacotherapy some studies excluded patients using specific medication (for example, corticosteroids [35,38-40,46], opioids $[39,41]$, antidepressants $[39,40,45]$, or adrenoceptor antagonists $[29,40,45])$, or the intake of medication was regulated prior to and on the day of testing [39,41]. In addition, two studies performed subgroup analyses with patients on different medical regimes $[41,45]$.

\section{Stress paradigms}

The stressors included in this review were experimental manipulations of stressful experiences, either of a psychological, exercise and/or sensory nature, and lasted between one minute and two hours.

\section{Psychological stressors}

Ten of 16 studies used a psychological stressor, applied for one minute to two hours [29-32,36-38,40,41,43-45].

Cognitive stressors Seven studies used cognitive stressors [29-32,36-38,42,44], namely, the Stroop Color-Word Interference test $[29,30,38]$; a two-minute cognitive discrimination task [29,30]; the Attention and Concentration Test, Syndrome Short Test, computerized-controlled Reaction Time Task and three parts of the Hamburg Wechsler Adult Intelligence Test (comprehension, digit span, block design) [42,44]; Multiple Choice Word Fluency; and the Benton Visual Memory Scale [44]. Mental arithmetic was assessed in two studies, either with a oneor two-minute serial subtraction task [31,32], or with a two-minute paced auditory serial addition test performed while patients were tilted to a head-up-tilt of 64 degrees [36,37].

Psychosocial stressors Three studies used a psychosocial stressor, namely, a 10-minute public speaking task [40]; the Trier Social Stress Test consisting of a five-minute public speech and a five-minute public mental subtraction task [41]; or a ten-minute public speech (including a five-minute preparation period) on the topic 'Children in today's society' [45]. 
Table 1: Study sample characteristics

\begin{tabular}{|c|c|c|c|c|c|c|c|c|}
\hline \multirow[t]{2}{*}{ Study } & \multirow[t]{2}{*}{ Patient sample } & \multirow[t]{2}{*}{ Control sample } & \multicolumn{6}{|c|}{ Pharmacotherapy of patient sample } \\
\hline & & & NSAIDs & DMARDs & Cortisteroids & Biologicals & Other & $\begin{array}{l}\text { Pharmacotherapy } \\
\text { comments: }\end{array}$ \\
\hline Dekkers et al., 2001 [38] & $\begin{array}{l}N=29 \text { RA patients; } \\
\text { No exclusion criteria given }\end{array}$ & $\begin{array}{l}\mathrm{N}=30 \mathrm{HC} \\
\text { Age and sex matched; } \\
\text { Exclusion: chronic } \\
\text { disease, chronic pain, } \\
\text { heart problems, } \\
\text { hypertension }\end{array}$ & + & + & -1 & o & o & $\begin{array}{l}1 \text { patients taking oral } \\
\text { prednisone }(5-15 \mathrm{mg}) \text { or } \\
\text { corticosteroid injections }<3 \\
\text { months prior to study onset } \\
\text { excluded }\end{array}$ \\
\hline Edwards et al., 2009 [39] & $\begin{array}{l}\mathrm{N}=19 \text { RA patients; } \\
\text { Exclusion: history of myocardial infarction } \\
\text { or cardiovascular disease, peripheral } \\
\text { neuropathy, Raynaud syndrome, vasculitis, } \\
\text { peripheral vascular disease, other } \\
\text { autoimmune or rheumatic disorders, } \\
\text { current infection, recent history of } \\
\text { substance abuse or dependence, } \\
\text { pregnancy, mood or anxiety disorder }\end{array}$ & $\begin{array}{l}\mathrm{N}=21 \mathrm{HC} ; \\
\text { No age and sex } \\
\text { difference with patients; } \\
\text { Same exclusion criteria } \\
\text { as patients; }\end{array}$ & $++^{2}$ & + & - & + & -3 & $\begin{array}{l}224 \mathrm{~h} \text { prior to study onset } \\
\text { no NSAIDs intake; } \\
{ }^{3} \text { Opioids, antidepressants } \\
\text { excluded; } \\
\text { Stable medication regime } \\
\text { of }>1 \text { months }\end{array}$ \\
\hline $\begin{array}{l}\text { Geenen et al., 1996/ } \\
1998[29,30]\end{array}$ & $\begin{array}{l}\mathrm{N}=21 \mathrm{RA} \text { patients; } \\
\text { Exclusion: other serious diseases }\end{array}$ & $\begin{array}{l}\mathrm{N}=20 \mathrm{HC} \\
\text { Age and sex matched; } \\
\text { Exclusion: chronic pain, } \\
\text { cardiovascular } \\
\text { complaints, chronic } \\
\text { diseases }\end{array}$ & + & + & o & o & -4 & $\begin{array}{l}{ }^{4} \alpha \text { and } \beta \text { adrenoceptor } \\
\text { antagonists excluded (only } \\
\text { for autonomic response } \\
\text { evaluation) }\end{array}$ \\
\hline $\begin{array}{l}\text { Hinrichsen et al., } 1989 \\
\text { [43] }\end{array}$ & $\begin{array}{l}\mathrm{N}=14 \text { SLE patients; } \\
\text { No exclusion criteria given }\end{array}$ & $\begin{array}{l}\mathrm{N}=14 \mathrm{HC}, \mathrm{N}=12 \\
\text { sarcoidosis patients; } \\
\text { Age and sex matched; }\end{array}$ & o & o & $+^{5}$ & o & o & $\begin{array}{l}524 \mathrm{~h} \text { prior to study onset } \\
\text { no corticosteroid therapy; }\end{array}$ \\
\hline $\begin{array}{l}\text { Hinrichsen et al., } 1992 \\
\text { [44] }\end{array}$ & $\begin{array}{l}\mathrm{N}=14 \text { SLE patients; } \\
\text { No exclusion criteria given }\end{array}$ & $\begin{array}{l}\mathrm{N}=14 \mathrm{HC}, \mathrm{N}=10 \mathrm{HC} \\
\text { taking corticosteroids; } \\
\text { Age and sex matched }\end{array}$ & o & o & +6 & o & o & $\begin{array}{l}64-10 \mathrm{mg} ; 24 \mathrm{~h} \text { prior to } \\
\text { study onset no } \\
\text { corticosteroid therapy }\end{array}$ \\
\hline
\end{tabular}




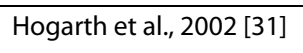
Remark: some patients have comorbid

Sjogren syndrome, Raynaud's

phenomenon, history of cerebral lupus

Jscobs et al., 2001 [40]

$\mathrm{N}=9$ RA patients, $\mathrm{N}=7$ SLE patients;

Exclusion: significant cardiovascular

diseases, concurrent infections, history of

other autoimmune disorders, drug or

alcohol abuse; no exacerbation of disease

4 weeks

Kurtais et al., 2006 [34]

\section{= 19 RA patients;}

Exclusion: severe illness, other general

contraindications for graded exercise test

Motivala et al., 2008 [41] N $=21$ RA patients;

Exclusion: cardiovascular disease

endocrine-related other immune disorders,

acute or chronic infections, pregnancy,

current psychiatric mood or anxiety

disorder

Palm et al., 1992 [42]

$\mathrm{N}=18$ RA patients;

No exclusion criteria given

\section{$\mathrm{N}=23 \mathrm{HC}$}

Age and sex matched;

Exclusion: diabetes

mellitus, hypertension

$\mathrm{N}=15 \mathrm{HC}$;

Age and sex matched;

Same exclusion criteria

as patients

$\mathrm{N}=14 \mathrm{HC}$

Age and sex matched;

Inclusion: sedentary

Exclusion: chronic

systemic disease,

chronic pain,

contraindication for

exercise test

\section{$\mathrm{N}=20 \mathrm{HC}$}

Age and sex matched;

Same exclusion criteria

as patients

\section{$\mathrm{N}=14 \mathrm{HC}$;}

Age and sex matched;

Half of all controls had

been hospitalized

because of coronary

heart disease or

hypertension
71-30 mg;

${ }^{8}$ Antihypertensive

medication $<1$ week prior

to study excluded

${ }^{9}$ adrenoceptor antagonists,

antidepressants and

benzodiazepines taken $<4$

weeks prior to study onset

excluded

07.5-15 mg;

$1124 \mathrm{~h}$ prior to study onset no NSAIDs taken;

$12<10 \mathrm{mg}$ (> $10 \mathrm{mg}$

excluded);

13 Opioids, oral

contraception excluded;

Stable medication regime

$>2$ months

$142,5-10 \mathrm{mg}$ 
Table 1: Study sample characteristics (Continued)

\begin{tabular}{|c|c|c|c|c|c|c|c|c|}
\hline Pawlak et al., 1999 [45] & $\begin{array}{l}\mathrm{N}=15 \text { SLE patients ( } \mathrm{N}<10 \text { for subanalyses: } \\
\text { NK cytotoxicity and \# } \beta \text {-adrenoceptors); } \\
\text { Exclusion: significant cardiovascular } \\
\text { diseases, concurrent infections, history of } \\
\text { other autoimmune disorders, drug or } \\
\text { alcohol abuse }\end{array}$ & $\begin{array}{l}\mathrm{N}=15 \mathrm{HC}(\mathrm{N}<10 \text { for } \\
\text { subanalyses: } \mathrm{NK} \\
\text { cytotoxicity and \# } \beta- \\
\text { adrenoceptors); } \\
\text { Age and sex matched; } \\
\text { Same exclusion criteria } \\
\text { as patients }\end{array}$ & o & + & +15 & o & -16 & $\begin{array}{l}15 \text { 5-10 mg; } \\
{ }^{16} \text { Adrenoceptor } \\
\text { antagonists, } \\
\text { antidepressants, } \\
\text { benzodiazepines taken }<4 \\
\text { week prior to study onset } \\
\text { excluded }\end{array}$ \\
\hline Perry et al., 1989 [32] & $\begin{array}{l}\mathrm{N}=19 \text { heterogeneous group of arthritis } \\
\text { patients (RA, psoriatic arthritis, ankylosing } \\
\text { spondylitis, fibrositis); } \\
\text { No exclusion criteria given }\end{array}$ & $\begin{array}{l}\mathrm{N}=38 \mathrm{HC}, 17 \text { patients } \\
\text { with myofascial pain; } \\
\text { No exclusion criteria } \\
\text { given }\end{array}$ & + & o & $\mathrm{O}$ & o & $+{ }^{17}$ & $\begin{array}{l}17 \text { Benzodiazepines, } \\
\text { psychotropics, and other } \\
\text { medication affecting ANS, } \\
\text { etc; } 12 \mathrm{~h} \text { prior to onset } \\
\text { study no medication taken } \\
\text { known to affect ANS }\end{array}$ \\
\hline Pool et al., 2004 [35] & $\begin{array}{l}\mathrm{N}=7 \text { RA patients, } \mathrm{N}=6 \mathrm{SLE} \text { patients; } \\
\text { No exclusion criteria given }\end{array}$ & $\begin{array}{l}\mathrm{N}=10 \mathrm{HC} ; \\
\mathrm{HC} \text { recruited from } \\
\text { hospital staff; } \\
\text { No exclusion criteria } \\
\text { given }\end{array}$ & + & + & - & o & -18 & $\begin{array}{l}18 \text { Phenothiazines (e.o. } \\
\text { drugs influencing PRL } \\
\text { levels) excluded }\end{array}$ \\
\hline Roupe et al., 2000 [46] & $\begin{array}{l}\mathrm{N}=20 \mathrm{JIA} \text { patients ( } \mathrm{N}=15 \text { for in vivo } \\
\text { analyses); } \\
\text { No exclusion criteria given }\end{array}$ & $\begin{array}{l}\mathrm{N}=20 \mathrm{HC}(\mathrm{N}=14 \text { for in } \\
\text { vivo analyses); } \\
\text { Age and sex matched; }\end{array}$ & + & + & - & o & o & \\
\hline Shalimar et al., 2006 [33] & $\begin{array}{l}\mathrm{N}=51 \text { SLE patients; } \\
\text { No exclusion criteria given }\end{array}$ & $\begin{array}{l}\mathrm{N}=30 \mathrm{HC} \\
\text { Age and sex matched }\end{array}$ & o & o & $\mathrm{O}$ & o & -19 & $\begin{array}{l}19 \text { Medication known to } \\
\text { affect HR, BP excluded }\end{array}$ \\
\hline $\begin{array}{l}\text { Veldhuijzen et al., 2005/ } \\
2008[36,37]\end{array}$ & $\begin{array}{l}\mathrm{N}=21 \mathrm{RA} \text { patients; } \\
\text { Exclusion: previously confirmed acute } \\
\text { coronary syndrome, } \mathrm{DM} \text {, serious psychiatric } \\
\text { diseases }\end{array}$ & $\begin{array}{l}\mathrm{N}=10 \text { OA patients; } \\
\text { Same exclusion criteria } \\
\text { as patients }\end{array}$ & + & + & + & + & +20 & $\begin{array}{l}{ }^{20} \text { Analgesics included; no } \\
\text { use of oral contraception }\end{array}$ \\
\hline
\end{tabular}

$\mathrm{RA}=$ rheumatoid arthritis, $\mathrm{SLE}=$ systemic lupus erythematosus, $\mathrm{HC}=$ healthy controls, JIA = juvenile idiopathic arthritis, $\mathrm{OA}=$ osteoarthritis, $\mathrm{DM}=$ diabetes mellitus. $+=$ Included; $-=$ Excluded; $\mathrm{O}=$ Not mentioned in article. ANS $=$ autonomic nervous system, BP = blood pressure, DMARDs = disease-modifying antirheumatic drugs, $\mathrm{HR}=$ heart rate, $\mathrm{NSAIDs}=$ nonsteroidal anti-inflammatory drugs, $\mathrm{PRL}=$ prolactin 


\section{Exercise stressors}

Three studies used exercise stressors, namely, a bicycle ergometer task, which involved cycling at a rate of $50 \mathrm{rev-}$ olutions per minute against a resistance of 12.5 watt for three minutes [38]; or an ergospirometric exercise test on a treadmill to the limit of subject's tolerance for several minutes $[34,35]$.

\section{Sensory stressors}

Eight studies assessed sensory stressors, most of which used thermal stimuli, usually experienced as painful. Five studies assessed responses to a cold stressor $[31,33,38,39,46]$, in which participants either had to put their hand in a bowl filled with (ice) cold water for several minutes $[38,39,46]$, or endure an ice-pack on their hand [31]. One study did not describe the cold pressor task [33]. Heat pulses [39], an acoustic stress test (several minutes) $[42,43]$ and a pupillary light flash test [32] were also used.

\section{Outcome measures}

Outcome measures were self-reported distress, autonomous nervous system responses, neuroendocrine responses, or immune responses (see Tables 2, 3 and 4).

\section{Self-reported distress}

Stress-induced self-reported distress was assessed in only two of 16 studies [41,45]. Subjective distress levels were either measured on a visual analogue scale (VAS) ranging from 0 to 100 [41] or on a VAS tension and excitement together with the State Trait Anxiety Inventory (STAI) [45].

\section{Measures of the autonomous nervous system}

Eleven studies assessed ANS responses (see Table 2) [29,31-33,37,40-43,45,46], namely, heart rate $[29,31,32,37,41,45]$, diastolic and/or systolic blood pressure $[29,31,33,41,45]$, mean arterial pressure, systemic vascular resistance, plasma volume and cardiac output $[36,37]$, and pre-ejection period (PEP) [41]. Furthermore, plasma (nor)epinephrine levels $[31,40,42,44-46]$ and skin conductance $[29,32]$ were assessed.

\section{Endocrine measures}

Eight studies assessed neuroendocrine responses (see Table 3) $[30,34,35,38,39,41,42,44]$, namely plasma $[30,34,38,41,42,44]$ or serum $[35,39]$ cortisol levels and plasma ACTH levels $[34,38,41]$. Two studies measured hormones other than those involved in the HPA axis, namely, plasma growth hormone $(\mathrm{GH})$ and insulin-like growth factor I (IGF-I) levels [34] and serum prolactin levels [35].

\section{Immunological measures}

Twelve studies assessed immune responses (see Table 4) $[30,35,36,38-46]$, namely, the total number of leucocytes [36,40,42-45], and the number of lymphocytes and/or subsets of lymphocytes, including B cells, T cells and NK cells $[30,35,40,42-45]$. Two studies assessed NK cell cyto- toxicity $[40,45]$. Six studies reported on cytokine levels [38-42,46], namely, plasma levels of cytokine IL-1 $\beta$ [42], IL-6 $[39,41,42]$ and TNF- $\alpha$ [39], ex vivo stimulated mononuclear cell production of IL-2 [40], IL-4 [38,40], IL-6 $[40,41,46]$, IL-8 [46], IL-10 [40], interferon (IFN)- $\gamma$ $[38,40]$ and TNF- $\alpha[41]$, or intracellular concentrations of IL-4, IL-6, IL-10 and IFN- $\gamma$ [40]. Other inflammation markers were also assessed, such as $C$-reactive protein (CRP) [36], the number of $\beta$-adrenoceptors on monocytes [45], $\beta$-adrenoceptor sensitivity [45], and plasma soluble IL-2 receptor levels [42].

\section{Time points of outcome measures}

All studies reported baseline values for the outcome measures, and all, except two [32,34], reported a resting period of 3 to 45 minutes. During administration of the experimental stressor, physiological reactivity was either assessed at specific time points (in three studies) $[35,41,42]$, or continuously throughout the stress procedure (only for autonomic measures such as heart rate, blood pressure and/or skin conductance; in four studies) $[29,32,37,45]$. All studies reported immediate post stress measurements, except two studies that only assessed stress reactivity at 30 minutes [34] or 60 minutes [35] after cessation of the stressor. Eight of 16 studies have one $[29,30,36-38,40,45]$ or more $[39,41,46]$ additional followup measurement points, ranging from 5 to 60 minutes after cessation of the stressor.

\section{Baseline characteristics}

Autonomic, neuroendocrine, and immune functions were assessed at baseline in patients with rheumatic disorders and controls. Results for the separate outcome measures are summarized in Tables 2, 3 and 4 .

\section{ANS variables}

ANS function at baseline was not different between patients with rheumatic disorders and controls in most studies. Cardiovascular variables, skin conductance, and catecholamine levels did not differ in nine studies $[29,31,36,37,40-42,44-46]$, whereas three studies reported significantly higher levels of autonomic activity at baseline (heart rate and pupil area [32]; epinephrine levels [46]) or lower levels ((nor)epinephrine [31]) in patients with rheumatic disorders compared with controls (see Table 2).

\section{Neuroendocrine variables}

Neuroendocrine function at baseline was not significantly different between patients with rheumatic disorders and controls. Five of eight studies reported similar baseline levels of cortisol, ACTH, GH, IGF-I, and prolactin $[34,35,39,41,42]$. Three studies reported higher levels of cortisol at baseline (in patients with RA) [30,38] or lower levels (in patients with SLE) [44] than in controls (see Table 3). Lower cortisol levels at baseline were also 
Table 2: Autonomic function in patients with systemic inflammatory rheumatic diseases

\begin{tabular}{|c|c|c|c|c|c|c|c|}
\hline \multirow{2}{*}{$\begin{array}{l}\text { Parameter } \\
\text { Heart rate }\end{array}$} & \multirow{2}{*}{$\begin{array}{l}\text { Studies \& patients (N) } \\
\text { [29] } 21 \text { RA vs. } 20 \mathrm{HC}\end{array}$} & \multicolumn{2}{|c|}{ Baseline patients vs. controls } & \multicolumn{2}{|c|}{ Stress reactivity within patients } & \multicolumn{2}{|c|}{ Stress reactivity patients vs. controls } \\
\hline & & RA: & $\begin{array}{l}\text { No difference } \\
{[29,36,37,41]}\end{array}$ & RA: & Increase $[29,36,37,41]$ & RA: & No difference $[36,37,41]$ \\
\hline & [41] 21 RA vs. $20 \mathrm{HC}$ & & & & & & Altered $(\downarrow)[29]$ \\
\hline & {$[36,37] 21$ RA vs. $10 \mathrm{OA}$} & SLE: & No difference $[31,45]$ & SLE: & Increase [45] & SLE: & No difference [31] (cogn.) [45] \\
\hline & [31] 23 SLE vs. $23 \mathrm{HC}$ & & & & Not reported [31] & & Altered ( $\downarrow)$ (cold) [31] \\
\hline & [45] 15 SLE vs. $15 \mathrm{HC}$ & Arthr: & Altered $(\uparrow)[32]$ & Arthr: & Increase [32] & Arthr: & No difference [32] \\
\hline & [32] 19Arthr vs. 38 HC, 17 MFP & & & & & & \\
\hline \multirow{6}{*}{$\begin{array}{l}\text { Blood pressure (diastolic/ } \\
\text { systolic) }\end{array}$} & [29] 21 RA vs. $20 \mathrm{HC}$ & RA: & No difference $[29,36,41]$ & RA: & Increase $[29,36,41]$ & RA: & No difference $[36,41]$ \\
\hline & [41] $21 \mathrm{RA}$ vs. $20 \mathrm{HC}$ & & & & & & Altered $(\downarrow)[29] /(\uparrow)[41]$ \\
\hline & [36] 21 RA vs. $10 \mathrm{OA}$ & SLE: & No difference $[31,45]$ & SLE: & Increase [45] & SLE: & No difference $[31,33,45]$ \\
\hline & [31] 23 SLE vs. $23 \mathrm{HC}$ & & Not reported [33] & & Not reported $[31,33]$ & & \\
\hline & [45] 15 SLE vs. $15 \mathrm{HC}$ & & & & & & \\
\hline & [33] 51 SLE vs. $30 \mathrm{HC}$ & & & & & & \\
\hline Mean arterial pressure (MAP) & [37] 21 RA vs. $10 \mathrm{OA}$ & RA: & No difference & RA: & Increase & RA: & No difference \\
\hline $\begin{array}{l}\text { Systemic vascular resistance } \\
\text { (SVR) }\end{array}$ & [37] 21 RA vs. $10 \mathrm{OA}$ & RA: & No difference & RA: & Increase severe subgroup & RA: & Altered $(\uparrow)$ severe subgroup \\
\hline Plasma volume (PV) & [36] 21 RA vs. $10 \mathrm{OA}$ & RA: & No difference & RA: & Decrease & RA: & No difference \\
\hline Cardiac output (CO) & [37] 21 RA vs. $10 \mathrm{OA}$ & RA: & No difference & RA: & No response & RA: & No difference \\
\hline Pre-ejection period (PEP) & [41] $21 \mathrm{RA}$ vs. $20 \mathrm{HC}$ & RA: & No difference & RA: & Decrease & RA: & No difference \\
\hline \multirow{3}{*}{$\begin{array}{l}\text { Plasma catecholamines } \\
\text { (nor)epinephrine }\end{array}$} & [42] 18 RA vs. $14 \mathrm{HC}$ & RA: & No difference $[40,42]^{*}$ & RA: & Increase [40] & RA: & No difference $[40,42]$ \\
\hline & [40] 9 RA, 7 SLE vs. $15 \mathrm{HC}$ & & & & No response [42] & & \\
\hline & [44] 14 SLE vs. $14 \mathrm{HC}, 10 \mathrm{HC}$ & SLE: & No difference $[40,45]$ & SLE: & Increase $[40,44,45]$ & SLE: & No difference $[40,45,44]^{*}$ \\
\hline
\end{tabular}


Table 2: Autonomic function in patients with systemic inflammatory rheumatic diseases (Continued)

\begin{tabular}{|c|c|c|c|c|c|c|c|}
\hline & [31] 23 SLE vs. $23 \mathrm{HC}$ & & Altered $(\downarrow)[31]$ & & & & \\
\hline & [45] 15 SLE vs. $15 \mathrm{HC}$ & & Not reported [44] & & & & \\
\hline & [46] $15 \mathrm{JIA}$ vs. $14 \mathrm{HC}$ & JIA: & No difference (NE) [46] & JIA: & Increase (NE)[46] & $\mathrm{JIA}:$ & No difference [46] \\
\hline & & & Altered $(\uparrow)(\mathrm{EPI})[46]$ & & No response (EPI)[46] & & \\
\hline \multirow[t]{2}{*}{ Skin conductance (SC) } & [29] 21 RA vs. $20 \mathrm{HC}$ & RA: & No difference [29] & RA: & Increase [29] & RA: & Altered $(\downarrow)[29]$ \\
\hline & [32] 19 Arthr vs. $38 \mathrm{HC}, 17 \mathrm{MFP}$ & Arthr: & No difference [32] & Arthr: & Increase [32] & Arthr: & Altered $(\uparrow)[32]$ \\
\hline Pupillary constriction & [32] 19 Arthr vs. $38 \mathrm{HC}, 17 \mathrm{MFP}$ & Arthr: & Altered $(\downarrow)$ & Arthr: & Not reported & Arthr: & Altered $(\downarrow)$ \\
\hline
\end{tabular}

* Findings assumed after inspection of descriptive data.

$=$ altered response pattern is more pronounced compared to a control group; = altered response pattern is diminished compared to a control group; $\mathrm{RA}=\mathrm{rheumatoid}$ arthritis, SLE = systemic lupus erythematosus, JIA = juvenile idiopathic arthritis, Arthr = heterogeneous group of arthritis patients, $\mathrm{HC}=$ healthy controls, OA = osteoarthritis, MFP = patients with myofascial pain,... NE = norepinephrine, $\mathrm{EPI}=$ epinephrine. 
Table 3: Neuroendocrine function in patients with systemic inflammatory rheumatic diseases

\begin{tabular}{|c|c|c|c|c|c|c|c|}
\hline \multirow{2}{*}{$\begin{array}{l}\text { Parameter } \\
\mathrm{ACTH}\end{array}$} & \multirow{2}{*}{$\begin{array}{l}\text { Studies \& patients (N) } \\
\text { [38] } 29 \text { RA vs. } 30 \text { HC } \\
\end{array}$} & \multicolumn{2}{|c|}{ Baseline patient vs. control } & \multicolumn{2}{|c|}{ Stress reactivity within patients } & \multicolumn{2}{|c|}{ Stress reactivity patients vs. controls } \\
\hline & & $\mathrm{RA}:$ & No difference $[34,38,41]$ & RA: & Increase $[34,41]$ & RA: & No difference $[34,38,41]$ \\
\hline & [34] 19 RA vs. $14 \mathrm{HC}$ & & & & Not reported [38] & & \\
\hline & [41] $21 \mathrm{RA}$ vs. $20 \mathrm{HC}$ & & & & & & \\
\hline \multirow[t]{8}{*}{ Cortisol } & [30] $21 \mathrm{RA}$ vs. $20 \mathrm{HC}$ & RA: & No difference $[34,35,39,41,42]$ & RA: & Decrease $[34,35,42]$ & RA: & No difference $[30,34,39,41,42]$ \\
\hline & [38] 29 RA vs. $30 \mathrm{HC}$ & & Altered $(\uparrow)[30,38]$ & & Change [30] & & Altered $(\downarrow)[35,38]$ \\
\hline & [39] 19 RA vs. $21 \mathrm{HC}$ & & No difference [35] & & Increase $[39,41]$ & & No difference [44] \\
\hline & [34] 19 RA vs. $14 \mathrm{HC}$ & SLE: & Altered $(\downarrow)[44]$ & SLE: & Decrease [35] & SLE: & Altered $(\downarrow)[35]$ \\
\hline & [41] 21 RA vs. $20 \mathrm{HC}$ & & & & No response [44] & & \\
\hline & [42] $18 \mathrm{RA}$ vs. $14 \mathrm{HC}$ & & & & & & \\
\hline & [35] $7 \mathrm{RA}, 6 \mathrm{SLE}$ vs. $10 \mathrm{HC}$ & & & & & & \\
\hline & [44] 14 SLE vs. $14 \mathrm{HC}, 10 \mathrm{HC}$ & & & & & & \\
\hline Growth hormone (GH) & [34] 19 RA vs. $14 \mathrm{HC}$ & RA: & No difference & RA: & Increase & RA: & No difference \\
\hline Insulin-like growth factor (IGF-I) & [34] 19 RA vs. $14 \mathrm{HC}$ & RA: & No difference & RA: & No response & RA: & No difference \\
\hline \multirow[t]{2}{*}{ Prolactin } & [35] 7 RA, 6 SLE vs. $10 \mathrm{HC}$ & RA: & No difference & RA: & No response & RA: & Altered $(\downarrow)$ \\
\hline & & SLE: & No difference & SLE: & No response & SLE: & Altered $(\downarrow)$ \\
\hline
\end{tabular}

$=$ altered response pattern is more pronounced compared to a control group; $=$ altered response pattern is diminished compared to a control group;

$\mathrm{RA}=$ rheumatoid arthritis, $\mathrm{SLE}=$ systemic lupus erythematosus, $\mathrm{HC}=$ healthy controls, $\mathrm{ACTH}=$ adrenocorticotropin hormone, 
Table 4: Immune function in patients with systemic inflammatory rheumatic diseases

\begin{tabular}{|c|c|c|c|c|c|c|c|}
\hline \multirow{2}{*}{$\begin{array}{l}\text { Parameter } \\
\text { Leucocytes }\end{array}$} & \multirow{2}{*}{$\begin{array}{l}\text { Studies \& patients (N) } \\
\text { [42] } 18 \text { RA vs. } 14 \text { HC }\end{array}$} & \multicolumn{2}{|c|}{ Baseline patient vs. control } & \multicolumn{2}{|c|}{ Stress reactivity within patients } & \multicolumn{2}{|c|}{ Stress reactivity patients vs. controls } \\
\hline & & & & & & & \\
\hline & [36] 21 RA vs. $10 \mathrm{OA}$ & RA: & No difference $[36,40,42]$ & RA: & Increase $[36,40,42]$ & RA: & No difference $[36,40,42]$ * \\
\hline & [40] 9 RA, 7 SLE vs. $15 \mathrm{HC}$ & SLE: & No difference $[43,45,44] *$ & SLE: & Increase [40,43-45] & SLE: & Altered $(\downarrow)[40,43,45]$ \\
\hline & [43] 14 SLE vs. $14 \mathrm{HC}, 12 \mathrm{SD}$ & & Altered $(\downarrow)[40]$ & & & & No difference [44] * \\
\hline & [44] $14 \mathrm{SLE}$ vs. $14 \mathrm{HC}, 10 \mathrm{HC}$ & & & & & & \\
\hline & [45] 15 SLE vs. $15 \mathrm{HC}$ & & & & & & \\
\hline \multirow[t]{6}{*}{ Total lymphocytes } & [30] $21 \mathrm{RA}$ vs. $20 \mathrm{HC}$ & RA: & Altered $(\downarrow)[30,42]$ & $\mathrm{RA}:$ & Increase $[30,40]$ & RA: & No difference $[30,40]$ \\
\hline & [42] 18 RA vs. $14 \mathrm{HC}$ & & No difference [40] & & No response [42] & & Altered $(\downarrow)[42]$ \\
\hline & [40] 9 RA, 7 SLE vs. $15 \mathrm{HC}$ & SLE: & Altered $(\downarrow)[40,43,45]$ & SLE: & Increase $[40,45]$ & SLE: & Altered $(\downarrow)$ [43-45] \\
\hline & [43] 14 SLE vs. $14 \mathrm{HC}, 12 \mathrm{SD}$ & & Not reported [44] & & No response[43,44] & & No difference [40] \\
\hline & [44] $14 \mathrm{SLE}$ vs. $14 \mathrm{HC}, 10 \mathrm{HC}$ & & & & & & \\
\hline & [45] 15 SLE vs. $15 \mathrm{HC}$ & & & & & & \\
\hline \multirow[t]{5}{*}{ Total T cells (CD3+) } & [30] $21 \mathrm{RA}$ vs. $20 \mathrm{HC}$ & RA: & No difference [40] & $\mathrm{RA}:$ & Increase [40] & RA: & No difference $[30,40]$ \\
\hline & [40] 9 RA, 7 SLE vs. $15 \mathrm{HC}$ & & Altered $(\downarrow)[30]$ & & No response [30] & & \\
\hline & [43] 14 SLE vs. $14 \mathrm{HC}, 12 \mathrm{SD}$ & SLE: & Altered $(\downarrow)[40,45]$ & SLE: & Increase $[40,45]$ & SLE: & No difference $[40,43]$ \\
\hline & [44] 14 SLE vs. $14 \mathrm{HC}, 10 \mathrm{HC}$ & & No difference $(\%)[43,44] *$ & & No response (\%) $[43,44]$ & & Altered $(\downarrow)$ [45]/(\%) [44] \\
\hline & [45]15 SLE vs. $15 \mathrm{HC}$ & & & & & & \\
\hline \multirow[t]{5}{*}{ Helper T cells (CD4+) } & [40] 9 RA, 7 SLE vs. $15 \mathrm{HC}$ & RA: & No difference $[35,40]$ & $\mathrm{RA}:$ & Increase [40] & RA: & Altered $(\downarrow)[35,40]$ \\
\hline & [35] 7 RA, 6 SLE vs. $10 \mathrm{HC}$ & & & & Decrease [35] & & \\
\hline & [43] 14 SLE vs. $14 \mathrm{HC}, 12 \mathrm{SD}$ & SLE: & Altered $(\downarrow)[40,45]$ & SLE: & No response $[40,45] /(\%)[44]$ & SLE: & Altered $(\downarrow)[45](\%)[43,44] /(\uparrow)$ \\
\hline & [44] 14 SLE vs. $14 \mathrm{HC}, 10 \mathrm{HC}$ & & No difference [35]/(\%) [43] & & Decrease $[35] /(\%)[43]$ & & [35] \\
\hline & [45] 15 SLE vs. $15 \mathrm{HC}$ & & Not reported [44] & & & & No difference [40] \\
\hline \multirow[t]{4}{*}{ Cytotoxic T cells (CD8+) } & [40] 9 RA, 7SLE vs. $15 \mathrm{HC}$ & RA: & No difference $[35,40]$ & RA: & Increase [40] & RA: & No difference [40] \\
\hline & [35] 7 RA, 6 SLE vs. $10 \mathrm{HC}$ & & & & No response [35] & & Altered $(\downarrow)[35]$ \\
\hline & [43] 14 SLE vs. $14 \mathrm{HC}, 12 \mathrm{SD}$ & SLE: & Altered $(\downarrow)[40,45]$ & SLE: & Increase $[40,45] /(\%)[43,44]$ & SLE: & No difference $[40,45,44] *$ \\
\hline & [44] 14 SLE vs. $14 \mathrm{HC}, 10 \mathrm{HC}$ & & No difference [35]/(\%) [43] & & Decrease [35] & & Altered $(\downarrow)[35] /(\%)[43]$ \\
\hline
\end{tabular}




\begin{tabular}{|c|c|c|c|c|c|c|c|}
\hline & [45] 15 SLE vs. $15 \mathrm{HC}$ & & Not reported [44] & & & & \\
\hline \multirow[t]{3}{*}{ B cells (CD19+) } & [30] $21 \mathrm{RA}$ vs. $20 \mathrm{HC}$ & RA: & Altered $(\downarrow)[30]$ & RA: & Increase [30] & RA: & No difference [30] \\
\hline & [43] 14 SLE vs. $14 \mathrm{HC}, 12 \mathrm{SD}$ & SLE: & Altered ( $\uparrow)(\%)[43]$ & SLE: & Increase (\%) [43] & SLE: & Altered $(\downarrow)(\%)[43,44]$ \\
\hline & [44] 14 SLE vs. $14 \mathrm{HC}, 10 \mathrm{HC}$ & & No difference (\%) [44]* & & No response (\%) [44] & & \\
\hline \multirow[t]{4}{*}{ NK cells (CD56+) } & [30] $21 \mathrm{RA}$ vs. $20 \mathrm{HC}$ & RA: & No difference [40] & RA: & Increase $[30,40]$ & RA: & No difference $[30,40]$ \\
\hline & [40] 9 RA, 7 SLE vs. $15 \mathrm{HC}$ & & Altered $(\downarrow)[30]$ & & & & \\
\hline & [44] 14 SLE vs. $14 \mathrm{HC}, 10 \mathrm{HC}$ & SLE: & Altered $(\downarrow)[40,45]$ & SLE: & Increase $[40,45]$ & SLE: & Altered $(\downarrow)[40,45]$ \\
\hline & [45] 15 SLE vs. $15 \mathrm{HC}$ & & Not reported [44] & & No response [44] & & No difference [44] \\
\hline \multirow[t]{2}{*}{ NK cell cytotoxicity } & [40] 9 RA, 7 SLE vs. $15 \mathrm{HC}$ & RA: & No difference [40] & RA: & No response [40] & RA: & Altered $(\downarrow)[40]$ \\
\hline & [45] 4 SLE vs. 8 HC & SLE: & No difference $[40,45]$ & SLE: & No response $[40,45]$ & SLE: & Altered $(\downarrow)[40,45]$ \\
\hline \multicolumn{8}{|l|}{ Cytokines } \\
\hline \multirow[t]{5}{*}{ IL-6 } & [39] 19 RA vs. $21 \mathrm{HC}$ & RA: & No difference $[40,41]$ & RA: & No response [40] & RA: & No difference [39-41] \\
\hline & [41] $21 \mathrm{RA}$ vs. $20 \mathrm{HC}$ & & Altered $(\uparrow)$ [39] & & Increase [39] & & \\
\hline & [42] 18 RA vs. $14 \mathrm{HC}$ & & & & Decrease (not plasma) [41] & & \\
\hline & [40] 9 RA, 7 SLE vs. $15 \mathrm{HC}$ & SLE: & No difference [40] & SLE: & No response [40] & SLE: & No difference [40] \\
\hline & [46] 15 JIA vs. $14 \mathrm{HC}$ & JIA: & Altered $(\uparrow)[46]$ & JIA: & Increase [46] & JIA: & Altered $(\uparrow)[46]$ \\
\hline \multirow[t]{2}{*}{ IL-2 } & [40] 9 RA, 7 SLE vs. $15 \mathrm{HC}$ & RA: & No difference & RA: & No response & RA: & No difference \\
\hline & & SLE: & No difference & SLE: & No response & SLE: & No difference \\
\hline \multirow[t]{2}{*}{ IL-4 } & [38] 29 RA vs. $30 \mathrm{HC}$ & RA: & No difference $[38,40]$ & RA: & No response [40] & RA: & No difference [40] \\
\hline & [40] 9 RA, 7 SLE vs. $15 \mathrm{HC}$ & SLE: & No difference [40] & SLE: & Increase [40] & SLE: & Altered $(\uparrow)[40]$ \\
\hline IL-8 & [46] 15 JIA vs. $14 \mathrm{HC}$ & JIA: & Altered $(\uparrow)$ & JIA: & No response & JIA: & No difference \\
\hline \multirow[t]{2}{*}{ IL-10 } & [40] 9 RA, 7 SLE vs. 15 HC & RA: & Altered $(\downarrow)$ (not intracell.) & RA & No response & RA: & No difference \\
\hline & & SLE: & Altered $(\downarrow)$ (not intracell.) & SLE: & No response & SLE: & No difference \\
\hline \multirow[t]{3}{*}{ IFN- $\gamma$} & [38] 29 RA vs. $30 \mathrm{HC}$ & RA: & No difference [38] & RA: & No response [40] & RA: & Altered $(\downarrow)[40]$ \\
\hline & [40] 9 RA, 7 SLE vs. $15 \mathrm{HC}$ & & Altered $(\downarrow)$ (not intracell.) [40] & & & & \\
\hline & & SLE: & Altered $(\downarrow)($ not intracell.) [40] & SLE: & No response [40] & SLE: & Altered $(\downarrow)[40]$ \\
\hline TNF- $a$ & [39] 19 RA vs. $21 \mathrm{HC}$ & RA: & No difference $[39,41]$ & RA: & Increase $[39,41]$ & RA: & Altered $(\uparrow)[39,41]$ \\
\hline
\end{tabular}


Table 4: Immune function in patients with systemic inflammatory rheumatic diseases (Continued)

\begin{tabular}{|c|c|c|c|c|c|c|c|}
\hline & [41] 21 RA vs. $20 \mathrm{HC}$ & & & & & & \\
\hline$\beta$-adenoceptors & [45] 7 SLE vs. $8 \mathrm{HC}$ & SLE: & No difference & SLE: & No response & SLE: & Altered $(\downarrow)$ \\
\hline$\beta$-adrenoceptor sensitivity & [45] 7 SLE vs. $8 \mathrm{HC}$ & SLE: & Altered $(\downarrow)$ & \multicolumn{2}{|c|}{ Not assessed } & \multicolumn{2}{|c|}{ Not assessed } \\
\hline sIL-2 receptor & [42] 18 RA vs. $14 \mathrm{HC}$ & RA: & Altered $(\uparrow)$ & RA: & No response & RA: & No difference \\
\hline C-reactive protein (CRP) & [36] 21 RA vs. 10 OA & RA: & No difference & RA: & Increase & RA: & Altered $(\uparrow)$ \\
\hline
\end{tabular}

* Findings assumed after inspection of descriptive data.

$=$ altered response pattern is more pronounced compared to a control group; = altered response pattern is diminished compared to a control group;

$\mathrm{RA}=$ rheumatoid arthritis, SLE = systemic lupus erythematosus, JIA = juvenile idiopathic arthritis, $\mathrm{HC}=$ healthy controls, $\mathrm{OA}=\mathrm{osteoarthritis,} \mathrm{SD}=$ sarcoidosis patients. IL $=$ interleukin, IFN- $\gamma=$ interferon- $\gamma$, TNF- $a=$ tumor necrosis factor $a$, sIL-2 receptor $=$ soluble interleukin-2 receptor, intracell. = intracellular interleukin concentration on the single-cell level 
observed in one study in which control subjects were taking corticosteroids [44].

\section{Immune variables}

Baseline leucocyte counts [36,40,42] were not different between patients with RA and controls. However, two of three studies found patients with RA to have lymphopenia at baseline [30,42]. Levels of cytokines and other inflammatory factors (IL-2, IL-4, IL-6, IFN- $\gamma$, TNF- $\alpha$, and CRP) were similar in patients with RA and controls in five studies [36,38-41]. Three studies reported higher baseline levels of IL-6 [39] and soluble IL-2 receptor [42] and lower basal levels of IL-10 and IFN- $\gamma[40]$ in patients with RA compared with controls.

Baseline leucocyte counts were not significantly different between patients with SLE and controls in most (three of four) studies [43-45]. One study reported baseline leucopenia in patients with SLE [40]. All studies reported lower lymphocyte counts (including lower subsets of lymphocytes) in patients with SLE than in controls $[40,43,45]$. Despite this baseline lymphopenia, a higher percentage of B cells (from the total lymphocyte count) was found once [43]. Only one study reported similar numbers of helper $\mathrm{T}$ and cytotoxic $\mathrm{T}$ cells in patients with SLE and controls [35]. Cytokine (IL-2, IL-4 and IL-6) levels at baseline were not significantly different between patients with SLE and controls [40]. However, cytokine levels of IL-10 and IFN- $\gamma$ at baseline and the sensitivity of $\beta$-adrenoceptors, which are involved in the transduction of autonomic signals to immune cells, were lower in patients with SLE than in controls [45]. The one study with immunological data on patients with JIA reported higher cytokine levels (IL-6 and IL-8) than in controls at baseline [46] (see Table 4).

In summary, at baseline autonomic function is similar in patients with RA and SLE and controls, with only limited evidence for heightened autonomic function in (a subgroup of) patients with rheumatic diseases. Neuroendocrine function at baseline is also comparable in patients with RA and SLE and controls, with only three studies reporting altered cortisol levels in patient groups. Specific immune variables, mainly (subsets of) lymphocyte counts, appear to be lower in patients with SLE than in controls.

\section{Psychophysiological responses to stress}

An overview of findings is given in Tables 2, 3 and 4.

\section{Self-reported distress}

Self-reported distress was increased significantly from baseline by psychosocial stress in patients with RA [41] and SLE [45] and in controls. This increase was greater in patients with RA [41] than in controls. This was not the case for patients with SLE [45].

\section{ANS variables}

The autonomic response to stress was assessed in patients with RA, SLE, JIA and in a heterogeneous group of patients with inflammatory arthritis. Results are summarized in Table 2. In patients with RA, experimental stress increased autonomic activity (heart rate $[29,36,37,41]$, blood pressure $[29,37,41]$, systemic vascular resistance [37], pre-ejection period (PEP) [41], plasma volume [36], skin conductance [29], and plasma (nor)epinephrine levels [40]), with the increase being similar to that seen in controls in most studies. However, three studies reported either diminished [29] or more pronounced [37,41] autonomic responses in (a subgroup of) patients. In patients with SLE, experimental stress increased autonomic activity (heart rate [45], blood pressure [45], and (nor)epinephrine levels $[40,44,45]$ ), the increase often being similar to that seen in controls $[31,33,40,45]$. Only one study observed a diminished autonomic response (heart rate), but only during one specific type of stressor (cold) [31]. The one study involving patients with JIA showed experimental stress to increase norepinephrine levels to a similar extent in patients and healthy controls [46]. In one study with a heterogeneous group of patients with inflammatory arthritis, experimental stress increased autonomic activity (heart rate, skin conductance, and pupillary constriction) [32], but this increase was smaller (pupillary constriction) or greater (galvanic response) than that of controls.

In summary, patients with rheumatic disorders respond to experimental stress with increased cardiovascular, galvanic and catecholamine responses. Whereas most autonomic responses to stress are similar to those of a control group, there is partial evidence (five studies) for altered stress-induced autonomic responses.

\section{Neuroendocrine variables}

Neuroendocrine reactivity, mainly measured as changes in cortisol levels, was assessed in patients with RA and SLE and in controls (see Table 3). Experimental stress elicited both an increase [39,41] and (more often) a decrease $[34,35,38,42]$ in cortisol levels in patients with RA. ACTH, which is activated upstream of cortisol, increased in response to stress [34,41]. In addition to the assessment of HPA axis hormones, one study also reported an increase in GH levels in response to a stressor [34]. The neuroendocrine response to stress of patients with RA was not significantly different from that of controls in most studies, but two studies reported that cortisol responses were diminished in patients with RA compared with controls [35,38]. In one study, experimental stress increased prolactin levels in controls but not in RA patients [35]. The effect of stress on neuroendocrine function in patients with SLE was inconsistent, with stress eliciting a decrease [35] or no change [44] in cortisol levels. A lack of responsiveness was also reported in 
Table 5: Autonomic (ANS), neuroendocrine (NE), and immune responses to different stressors in patients with systemic inflammatory rheumatic diseases

\begin{tabular}{|c|c|c|c|c|c|c|c|}
\hline \multirow[t]{2}{*}{ Stress paradigm } & \multirow[t]{2}{*}{ Studies } & \multicolumn{2}{|l|}{ ANS } & \multicolumn{2}{|c|}{ NE system } & \multicolumn{2}{|c|}{ Immune system } \\
\hline & & Measure & Response & Measure & Response & Measure & Response \\
\hline \multirow[t]{2}{*}{ Psychological stress } & & $\mathrm{HR}$ & $\uparrow \operatorname{RA}[29,37]$ & Cortisol & $\downarrow / \uparrow R A[30]$ & Leucocytes & $\uparrow R A[36]$ \\
\hline & & & $\uparrow \operatorname{Arthr}[32]$ & & 0 SLE[44] & & $\uparrow S L E[44]$ \\
\hline \multirow[t]{11}{*}{ Cognitive tasks } & {$[38]^{*}[29,30,44]$} & SC & $\uparrow R A[29]$ & & & Lymphocytes & $\uparrow R A[30]$ \\
\hline & {$[31]^{* *}[42]^{*}$} & & $\uparrow \operatorname{Arthr}[32]$ & & & & O SLE[44] \\
\hline & {$[32,36,37]$} & $\mathrm{CO}$ & $\uparrow R A[37]$ & & & $\mathrm{T}$ & $0 \mathrm{RA}[30]$ \\
\hline & & PV & $\uparrow R A[36]$ & & & & O SLE[44] \\
\hline & & SVR & $\uparrow R A[37]$ & & & B & $\uparrow R A[30]$ \\
\hline & & $\mathrm{BP}$ & $\uparrow \operatorname{RA}[29,36,37]$ & & & & O SLE[44] \\
\hline & & $\mathrm{CA}$ & $\uparrow S L E[44]$ & & & NK & $\uparrow R A[30]$ \\
\hline & & & & & & & O SLE[44] \\
\hline & & & & & & Th & O SLE[44] \\
\hline & & & & & & Tcyt & $\uparrow$ SLE[44] \\
\hline & & & & & & CRP & $\uparrow R A[36]$ \\
\hline Psychosocial & {$[40,41,45]$} & HR & $\uparrow R A[41]$ & Cortisol & $\uparrow R A[41]$ & Leucocytes & $\uparrow \mathrm{RA}[40]$ \\
\hline \multirow[t]{14}{*}{ tasks } & & & $\uparrow S L E[45]$ & АСTH & $\uparrow R A[41]$ & & $\uparrow \operatorname{SLE}[40,45]$ \\
\hline & & $\mathrm{BP}$ & $\uparrow R A[41]$ & & & Lymphocytes & $\uparrow \mathrm{RA}[40]$ \\
\hline & & PEP & $\downarrow R A[41]$ & & & & $\uparrow \operatorname{SLE}[40,45]$ \\
\hline & & $C A$ & $\uparrow R A[40]$ & & & $\mathrm{T}$ & $\uparrow R A[40]$ \\
\hline & & & $\uparrow \operatorname{SLE}[40,45]$ & & & & $\uparrow \operatorname{SLE}[40,45]$ \\
\hline & & & & & & Tcyt & $\uparrow \mathrm{RA}[40]$ \\
\hline & & & & & & & $\uparrow \operatorname{SLE}[40,45]$ \\
\hline & & & & & & NK & $\uparrow \mathrm{RA}[40]$ \\
\hline & & & & & & & $\uparrow \operatorname{SLE}[40,45]$ \\
\hline & & & & & & Th & $\uparrow \mathrm{RA}[40]$ \\
\hline & & & & & & & 0 SLE[40,45] \\
\hline & & & & & & NK toxicity & $0 \mathrm{RA}[40]$ \\
\hline & & & & & & & 0 SLE[ $[40,45]$ \\
\hline & & & & & & IL-6 & $\downarrow[41] / 0[40]$ RA \\
\hline
\end{tabular}




\begin{tabular}{|c|c|c|c|c|c|c|c|}
\hline & & & & & & & O SLE[40] \\
\hline & & & & & & \multirow[t]{2}{*}{ IL-4 } & $0 \mathrm{RA}[40]$ \\
\hline & & & & & & & $\uparrow \mathrm{SLE}[40]$ \\
\hline & & & & & & \multirow[t]{2}{*}{ IL-2 } & $0 \mathrm{RA}[40]$ \\
\hline & & & & & & & O SLE[40] \\
\hline & & & & & & \multirow[t]{2}{*}{ IL-10 } & $0 \mathrm{RA}[40]$ \\
\hline & & & & & & & O SLE[40] \\
\hline & & & & & & \multirow[t]{2}{*}{ IFN- $\gamma$} & $0 \mathrm{RA}[40]$ \\
\hline & & & & & & & O SLE[40] \\
\hline & & & & & & TNF-a & $\uparrow R A[41]$ \\
\hline & & & & & & $\beta-A R$ & O SLE[41] \\
\hline \multirow[t]{7}{*}{ Exercise } & \multirow[t]{7}{*}[38]{$^{*}[34,35]$} & & & Cortisol & $\downarrow R A[34,35]$ & \multirow[t]{2}{*}{ Th } & $\downarrow R A[35]$ \\
\hline & & & & & $\downarrow$ SLE[35] & & $\downarrow$ SLE[35] \\
\hline & & & & АCTH & $\uparrow \mathrm{RA}[34]$ & \multirow[t]{5}{*}{ Tcyt } & $0 \mathrm{RA}[35]$ \\
\hline & & & & $\mathrm{GH}$ & $\uparrow \mathrm{RA}[34]$ & & $\downarrow$ SLE[35] \\
\hline & & & & IGF-I & O RA[34] & & \\
\hline & & & & PRL & 0 RA[35] & & \\
\hline & & & & & O SLE[35] & & \\
\hline \multicolumn{8}{|l|}{ Sensory stress } \\
\hline \multirow[t]{3}{*}{ Cold induction } & {$[38]^{*}[39]$} & \multirow[t]{9}{*}{$\mathrm{NE}$} & \multirow[t]{9}{*}{$\uparrow J I A[46]$} & \multirow[t]{9}{*}{ Cortisol } & \multirow[t]{9}{*}{$\uparrow \mathrm{RA}[39]$} & Leucocytes & $\uparrow \operatorname{SLE}[43]$ \\
\hline & {$[31]^{* *}[46]$} & & & & & Lymphocytes & O SLE[43] \\
\hline & {$[33]^{* *}$} & & & & & $\mathrm{~T}$ & O SLE[43] \\
\hline Heat induction & [39] & & & & & Tcyt & $\uparrow \operatorname{SLE}[43]$ \\
\hline Acoustic stress & {$[43,42]^{*}$} & & & & & B & $\uparrow S L E[43]$ \\
\hline \multirow[t]{4}{*}{ Pupillary light } & {$[32]^{* *}$} & & & & & Th & $\downarrow$ SLE[43] \\
\hline & & & & & & \multirow[t]{2}{*}{ IL-6 } & $\uparrow \mathrm{RA}[39]$ \\
\hline & & & & & & & $\uparrow J I A[46]$ \\
\hline & & & & & & TNF-a & $\uparrow \mathrm{RA}[39]$ \\
\hline
\end{tabular}

$\uparrow=$ increase in response to stressor, $\downarrow=$ decrease in response to stressor, $0=$ no response to stressor. RA = rheumatoid arthritis, SLE $=$ systemic lupus erythematosus, JIA $=$ juvenile idiopathic arthritis, arthr $=$ heterogeneous group of patients with inflammatory arthritis. ${ }^{*}=$ study not described in Table because more than one stress paradigm was used ${ }^{* *}=$ study not described in Table due to lack of within-subject measurements. $\mathrm{HR}=$ heart rate, $\mathrm{SC}=$ skin conductance, $\mathrm{BP}=$ blood pressure, $\mathrm{NE}=$ norepinephrine, $\mathrm{CA}=$ catecholamines, $\mathrm{PC}=$ pupillary constriction, $\mathrm{PV}=$ plasma volume, $\mathrm{PEP}=$ pre-ejection period, $\mathrm{CO}=$ cardiac output, $\mathrm{SVR}=$ systemic vascular resistance, $\mathrm{C}=$ cortisol, $\mathrm{ACTH}=$ adrenocorticotropin hormone, $\mathrm{PRL}=\mathrm{prolactin}, \mathrm{GH}=$ growth hormone, $\beta-\mathrm{AR}=\beta$ adrenoceptor, IGF-I = insulin-like growth factor I, IFN- $\gamma=$ interferon- $\gamma$, TNF- $\alpha=$ tumor necrosis factor $\alpha, \mathrm{IL}=$ interleukin, CRP $=\mathrm{C}$-reactive protein, B = B cell, T $=$ T cell, Th $=$ helper T cell, Tcyt $=$ cytotoxic T cell, $\mathrm{NK}=$ natural killer cell. 
control subjects $[35,44]$. Experimental stress did not increase prolactin levels in patients with SLE [35].

In summary, although cortisol and ACTH levels change in response to experimental stress, differences between patients with rheumatic diseases (RA and SLE) and control groups have been reported in only two studies. There is preliminary evidence (one study) that the prolactin response to stress is different in patients than in controls.

\section{Immune variables}

Because several immune variables were used in the various studies to assess the effect of experimental stress on immune function, we have classified results on the basis of changes in leucocyte and lymphocyte counts and inflammatory markers (see Table 4).

\section{Leucocytes and (subgroups of) lymphocytes In}

patients with RA, experimental stress consistently increased the number of leucocytes [36,40,42], and increased the number of lymphocytes [30,40], with increases in the number of B cells [30], total T cells [40] and cytotoxic $\mathrm{T}$ cells [40] and either an increase [40] or a decrease [35] in helper $\mathrm{T}$ cells. The stress-induced increase in NK cell numbers $[30,40]$ did not result in an increase in NK cell activity [40]. Most studies did not detect a difference in the immune response (leucocyte and lymphocyte counts) to stress of patients with RA and controls, but one study reported lower lymphocyte counts after stress in patients with RA [42]. Changes compared with controls were noted for helper $\mathrm{T}$ cells $[35,40]$ and cytotoxic T cells [35]. In patients with SLE, stress induced a consistent increase in the number of leucocytes [40,43-45], and a less consistent increase in the number of lymphocytes $[40,45]$. Stress increased the number of total T cells $[40,45]$, number $[40,45]$ and percentage $[43,44]$ of cytotoxic $T$ cells, and the percentage of $B$ cells [43], and decreased the number of helper $T$ cells $[35,43]$. Again, although NK cell numbers increased after stress $[40,45]$, NK cell activity did not increase [40,45]. Comparison showed that the immune response to stress was often smaller in patients with SLE than in controls: the increase in total leucocyte counts in patients with SLE was smaller than that of controls $[40,43,45]$, as was the increase in total lymphocyte count [43-45]. Moreover, the stress-induced change in subsets of lymphocytes was often smaller for B cells $[43,44]$, total $\mathrm{T}$ cell numbers $[44,45]$, helper T cells [43-45], cytotoxic T cells [35,43], and NK cells $[40,45]$.

Thus, total leucocyte counts and lymphocyte subsets change in response to stress in patients with rheumatic diseases, with patients with SLE having a smaller response (in addition to their baseline lymphopenia) than controls; no consistent alterations were found for patients with RA. NK cell cytotoxicity is diminished in patients with RA and SLE compared with control.
Inflammatory markers In patients with RA, experimental stress did not change levels of various inflammatory markers (IL-2, soluble IL-2 receptor, IL-4, IL-10 and IFN$\gamma)[40,42]$, although levels of CRP [36] and TNF- $\alpha$ increased after stress [39,41]. Results for IL- 6 were inconsistent, with stress inducing a decrease [41], an increase [39] or no change [40] in levels. Patients differed from controls, having smaller IL-10 and IFN- $\gamma$ responses [40], and larger TNF- $\alpha[39,41]$ and CRP responses [36]. In patients with SLE, experimental stress did not change levels of cytokines and other inflammatory markers (IL-2, IL-6, IL-10, IFN- $\gamma, \beta$-adrenoceptors) [40,45], although levels of IL-4 increased after stress [40]. Patients with SLE differed from controls in their response to stress, having a larger increase in IL-4, smaller IL-10 and IFN- $\gamma$ responses [40], and fewer $\beta$-adrenoceptors on monocytes [40,45]. In patients with JIA, stress did not affect IL-8 production, but increased IL-6 production [46]. This increase was not observed in controls. Thus, although in general experimental stress does not seem to influence levels of certain cytokines and inflammatory markers in patients with rheumatic diseases (for example, sIL-2, IL-8, IL-10, IFN$\gamma, \beta$-adrenoceptors), it does cause specific changes in CRP and TNF- $\alpha$ in patients with RA, changes in IL-4 in patients with SLE, and changes in IL-6 in patients with JIA. These changes are not observed in controls.

In summary, the leucocyte and lymphocyte responses to stress are smaller in patients with SLE than in controls but consistent differences with controls are not seen in patients with RA. Experimental stress does not seem to affect NK cell cytotoxicity in either patient group. However, specific stress-induced changes in inflammatory markers are reported in patients with RA (CRP, TNF- $\alpha$ ), SLE (IL-4) and JIA (IL-6) that are not observed in controls.

\section{Physiological stress reactivity for specific types of stressors}

As seen above, experimental stress has a distinct effect on the autonomic, neuroendocrine, and immune systems. Below, we summarize the effect of the different types of experimental stress (cognitive, psychosocial, physical, and sensory), to determine whether different types of stress elicit different responses in different patients groups (see Table 5). Studies in which more than one type of stressor was used, were not included [38,42] unless outcome measures were reported separately for the different types of stressors [31,32].

\section{Cognitive stressors}

Five studies investigated a cognitive stressor in patients with RA, SLE, or inflammatory arthritis [29-32,36,37,44]. In all patient groups, cognitive stress consistently increased autonomic function: heart rate [29,32,37], blood pressure [29,36,37], skin conductance [29,32], cardiac output and systemic vascular resistance [37], plasma 
volume [36], and catecholamines [44]. Patients with RA and SLE had different cortisol responses to cognitive stress [30,44] (see Table 5). Cognitive stress elicited changes in leucocyte counts, lymphocyte counts, subsets of lymphocytes, and CRP levels in patients with RA $[30,36]$, but only changes in leucocyte counts and cytoxic T cell numbers in patients with SLE [44].

\section{Psychosocial stressors}

A psychosocial stressor was used in three studies of patients with RA and SLE [40,41,45]. Psychosocial stress consistently increased autonomic activity (heart rate $[41,45]$, blood pressure [41], pre-ejection period [41] and catecholamine levels $[40,45])$ in patients with RA and SLE and increased neuroendocrine variables (cortisol and ACTH [41]) in patients with RA. Some immune responses were similar in the two patient groups (leucocyte counts, lymphocyte counts, (cytotoxic) T cells, NK cells and NK cell cytoxicity $[40,45])$, whereas others were different (helper T cells [40], IL-4 [40]) (see Table 5). Cytokine levels were not influenced by psychosocial stress, with the exception of an increase in IL-4 in patients with SLE, an increase in TNF- $\alpha$ in patients with RA, and inconsistent findings for IL-6.

\section{Exercise stressors}

The stress of exercise was investigated in two studies with patients with RA and SLE $[34,35]$. These studies did not assess autonomic function; however, cortisol levels decreased in both groups of patients [34,35] whereas ACTH levels and GH levels increased [34]. Exercise stress elicited a different cytotoxic $\mathrm{T}$ cell response in patients with RA and SLE [35] (see Table 5).

\section{Sensory stressors}

Sensory stressors were used in six studies involving patients with SLE and JIA [31-33,39,43,46]. Sensory stress increased catecholamine levels [46] and cortisol levels [39]. It also increased leucocyte counts, changed percentages of certain subsets of lymphocytes [43], and increased levels of IL-6 [39,46] and TNF- $\alpha$ [39] (see Table 5).

In summary, although few data are available about the effects of specific types of stress, results suggest that psychosocial, cognitive and sensory stress consistently increase autonomic activity. Changes in neuroendocrine variables in response to psychosocial, cognitive and sensory stressors are observed in patients with RA only. Psychosocial stress seems to elicit the strongest immune response in all patient groups studied.

\section{Discussion}

A better understanding of the acute physiological response to stress in patients with inflammatory rheumatic diseases might further our knowledge of how stress affects the health of these patients. We reviewed the effects of time-limited stressors on autonomic, endocrine, and immune variables in patients with chronic inflammatory rheumatic diseases. Results suggest that autonomic and neuroendocrine responses to stress are not consistently altered in patients with rheumatic disorders compared with controls, although patients do appear to show a distinct immune response to stress. Psychosocial stress might prove to be the best tool to evaluate these specific immune responses to stress.

\section{Physiological stress response systems ANS}

Although previous studies that used regular autonomic function tests showed autonomic reactivity to be altered in different populations of patients with rheumatic diseases compared with controls [2,47-50], we failed to find consistent evidence for these alterations in studies investigating psychological, exercise, or sensory stress. The autonomic function of patients with rheumatic disorders was not only similar to that of controls at baseline but also after experimental stress, with cardiovascular, galvanic and catecholaminergic responses largely comparable to those of controls. Only a minority of studies reported autonomic function to be altered in these patients (as compared with controls) [29,31,32,37]. Studies have shown that alterations in autonomic function in response to stress are correlated with disease severity $[36,37,48,51]$, which suggests that only subgroups of patients with severe disease show distinct alterations in autonomic function. Changes in autonomic function might also be linked to physical deconditioning, vascular inflammation and accelerated atherosclerosis [52], which could explain the inconsistencies in the literature on this subject. It should also be noted that alterations in autonomic function might reflect a change in sympathetic or parasympathetic activity or in the balance of the two systems $[53,54]$. Autonomic measures used in experimental stress paradigms (for example, the heart rate response) do not always differentiate between these systems, in contrast to regular autonomic function tests [55], which specifically aim to distinguish between sympathetic dysfunction and parasympathetic neuropathy $[49,51,56]$.

\section{HPA axis}

The expected increase in neuroendocrine variables in response to a stressor was only observed in two studies with patients with RA [39,41]. This increase is consistent with previous studies reporting significant and consistent endocrine responses to psychosocial stress both in healthy subjects and in various patient populations without rheumatic disorders [24,25,41,57-60,60-62]. The change in cortisol levels is consistent with the endocrine response to real-life anticipation stress before hip or knee surgery in patients with RA $[63,64]$. However, most studies evaluated in this review reported a decrease in cortisol levels after stress, both in patients and controls, which might reflect the normal diurnal rhythmic decline in cor- 
tisol levels instead of a stress response [34,35,38,42]. All studies, except the two reporting an increase in cortisol levels, assessed patients in the early morning hours, when cortisol levels are known to decrease sharply.

Fuelling the ongoing debate on whether or not HPA axis responses are diminished in patients with rheumatic disease compared with healthy subjects, we did not find consistent changes in HPA axis function in response to psychological and exercise stress in patients with rheumatic diseases. Indeed, only two studies showed a different neuroendocrine response to stress in patients and controls [35,38]. Previous studies involving pharmacological stimulation of the HPA axis reported either similar or slightly lower responses in patients with rheumatic diseases compared with controls $[27,28]$. However, results should be interpreted with caution because stress manipulation may have been ineffective. Variations across studies in disease duration and activity, age, sex and sampling time might contribute to the variability in HPA axis responses in patients with rheumatic diseases. Moreover, attention should be paid to the role of pharmacotherapy (for example, corticosteroids), which could influence the endocrine response to stress [65].

\section{Immune system}

Most studies reported that total leucocyte counts and specific subsets of lymphocytes (not total number per se) increased after stress in patients with rheumatic diseases. The most consistent finding was an increase in the number of NK cells, as reported in earlier studies showing that the most robust effect of acute time-limited stressors in healthy subjects is a marked increase in the number of NK cells, which suggest an activation of innate immunity [15]. However, NK cell cytotoxicity after stress was lower in patients than in controls, possibly due to permanent activation as a consequence of the inflammatory disease $[40,45]$. The change in cytokine levels elicited by stress in patients with rheumatic disorders was small or inconsistent, for example, for the most frequently measured cytokine IL-6 [40,41,46]. Previous semi-experimental studies with patients with RA reported ambiguous results, reporting either significantly increased [63] or decreased levels of IL-6 [64] during anticipation of planned knee or hip arthroplasty. In addition, in a study evaluating responses to cryotherapy, patients with RA not treated with glucocorticoids responded with an increase in IL-6 levels [65]. A recent meta-analysis showed IL-6 levels to be consistently increased after psychosocial stress in healthy individuals [16]. The contradictory findings regarding cytokine responses in patients with rheumatic diseases might be due to methodological caveats, such as failed stress manipulation or small sample sizes (for example, in Jacobs et al. (2001)). However, it is also possible that cytokines do not effectively regulate immune function systemically, but instead near the effec- tor site [40]. Future studies involving patients with rheumatic diseases should measure local immune variables in samples obtained from joint tissue or synovial fluid $[66,67]$. Another explanation is that patients or subgroups of patients might have different cytokine responses (for example, TNF- $\alpha$ ) because of differences in disease severity [68] or pharmacotherapy $[41,65]$. In general, as could be expected for immune-mediated diseases, the immune response to stress was different in patients and controls, and also differed between the various types of inflammatory rheumatic diseases.

\section{Differences in type of stressor, rheumatic disease and (time points of) outcome measures}

Findings of this review should be interpreted with caution due to differences in the types of stress induced, the various rheumatic diseases evaluated, and the variability in outcome measures and assessment times. In addition, the highly variable methodological quality of the studies, the small number of studies, and the relatively small patient samples hinder an unequivocal interpretation of findings. Future research urgently requires well-designed studies with larger numbers of patients. These studies should, for example, systematically take into account physiological baseline levels.

\section{Stress paradigms}

Only the studies of psychosocial stress evaluated all three physiological stress response systems (autonomic, neuroendocrine and immune) and, moreover, consistently reported stress-induced increases at all three levels. This is in line with previous studies showing significant and consistent autonomic, neuroendocrine, and immune responses to psychosocial stress in both healthy subjects and patient groups [25,41,57-60,60-62,69]. In contrast, cognitive stressors did not consistently alter neuroendocrine variables, and immune responses were small in patients with SLE. The stress of exercise was found not to activate the HPA axis in patients with rheumatic diseases, in contrast to what has previously been observed in healthy subjects [70], although studies suggest that neuroendocrine changes are only observed after exercise of longer duration [71]. Data on immune responses were very limited. The studies that assessed sensory stress reported stress-induced increases in autonomic, neuroendocrine, and immune function, but data were limited. Because most sensory stressors are also frequently applied to induce pain [72,73], future studies of stress should take into account whether a stressor is paininducing in order to unravel the relationship between stressors, pain and physiological responses [74]. Overall, more research into the effects of psychosocial stress on physiological function is needed, especially because these stress paradigms seem to generate the strongest response at all three physiological levels and probably have the 
greatest ecological validity. Importantly, insight in physiological responses to acute stressors cannot automatically be translated to the field of daily stressors, major stress events or even chronic stressors [5,75]. For example, studies in healthy and other disease populations suggest that chronic stress might induce hypocortisolism $[6,76]$ and global immunosuppression of both innate and specific immunity [15]. Overall, there is a lack of studies examining the association between physiological parameters and chronic stressors in rheumatic populations [2]. Studies investigating stressors in daily life reported an association between stressors, mainly of an interpersonal nature, and concentrations of cytokines and lymphocytes (for example, IL-6, soluble IL-2 receptors and T cell numbers) [77-79]. However, results are inconclusive [80] and future studies are needed to elucidate how acute and chronic stressors differentially affect inflammatory rheumatic diseases.

\section{Disease and treatment characteristics}

In contrast to autonomic and neuroendocrine responses, the immune response elicited seemed to be specific to the type of inflammatory rheumatic disorder involved, and this was especially evident for psychosocial stress. Patients with SLE often have baseline lymphopenia and this review showed that stress led to diminished cell mobilization and consequently less pronounced stressinduced changes in lymphocyte (sub)populations in these patients compared with controls and patients with RA. In contrast, patients with RA did not consistently show these alterations, but only alterations in certain subpopulations of lymphocytes. Furthermore, the cytokine response to stress might be distinct for different rheumatic populations. The effect of pharmacotherapy (for example, corticosteroids and biologicals) on neuroendocrine and immune responses to stress in these populations is unclear. Only two of 16 studies conducted subgroup analyses to reveal possible effects of pharmacotherapy on response patterns. Motivala et al. (2008) found significant stress-induced increases in TNF- $\alpha$ only in patients with RA not treated with TNF- $\alpha$ antagonists (and no effect of corticosteroid treatment), and Pawlak et al. (1999) reported no significant difference in physiological variables between patients with SLE treated with corticosteroids and/or disease-modifying antirheumatic drugs (DMARDs) and non-treated patients [45]. Four other studies also suggest no effect of pharmacotherapy (more specifically, DMARDs [38], nonsteroidal antiinflammatory drugs (NSAIDs) [34,38], and corticosteroids $[34,43,44]$ ) on stress reactivity patterns based on descriptive data. The other studies do not mention possible effects of pharmacotherapy [29-33,35-37,39,40,42,46].

Clearly, patient samples are often too small to draw conclusions. More studies comparing different inflammatory diseases and healthy participant are needed to address the question whether the physiological response to stress is disease-specific, knowledge which might facilitate our understanding of factors contributing to the maintenance and exacerbation of rheumatic disorders.

\section{Outcome measures}

The heterogeneity of outcome measures, especially with regard to immune variables, makes it difficult to compare studies of the stress response. Moreover, stressors that activate the physiological stress system via the hypothalamus and subsequent down-stream cascades might induce alterations in hormones, peptides and cytokines other than discussed and assessed in this review (for examples, dehydroepiandosterone (sulfate) (DHEA-S), neuropeptide Y (NPY), substance P). Previous studies suggest that alterations in the stress response in patients with rheumatic disorders might be located in the interaction between the two stress axes (HPA and ANS) and the immune system, more specifically in the number or signalling capacity of $\beta$-adrenoceptors $[45,48,66,81]$ or glucocorticoid receptors [82-84] on lymphocytes.

Although the HPA axis, ANS, and immune system are thought to function in an interdependent fashion, only four of 16 studies in this review investigated outcome variables that evaluate all three stress response systems. Cardiovascular reactivity during a mental stress task has been shown to be associated with the subsequent cortisol response [25], and cardiovascular responses have been associated with post stress levels of cytokines such as IL-6 and TNF- $\alpha$ [85]. In addition, HPA axis variables are correlated with immune responses after stress [53]. Future studies should try to integrate the responses of different systems by simultaneously assessing (para)sympathetic responses, neuroendocrine variables, and immune factors, to increase our knowledge of the coordination and possible dysregulation of these systems [59].

\section{Psychological variables}

In addition to assessing the physiological response to experimental real-life stressors, it is important to consider other key elements of the stress response, such as the individual's appraisal of the threat of the event, perceived distress, coping behaviour and personality characteristics [4]. As the appraisal of threat might partly determine the biological stress response of an individual, self-reported measures of distress appear to be a simple and adequate manipulation check in studies of psychosocial stress. Furthermore, acute stress responses are known to be correlated with individual differences in psychological factors, such as coping styles [86], trait anxiety $[87,88]$, depressed mood [59,89,90], perfectionism [91], social inhibition [92] and anticipatory cognitive appraisal [93-96]. However, none of the studies, except one [74], assessed individual psychological characteristics. In addition, only two studies in this review assessed stressinduced self-reported distress $[41,42]$ and only three 
studies $[36,37,39,41]$ explicitly excluded affective disorders. Although half of all studies excluded patients with (severe or chronic) comorbidity, these were mainly physical in nature. We cannot rule out the possibility that patients with psychiatric disorders are included in these studies, obscuring the major research question whether inflammatory rheumatic diseases are related to a specific physiological stress responses profile. Future studies of stress responses in patients with rheumatic diseases should include individual psychological characteristics (for example, personality, coping styles) and affective responses to help identify whether patients differ with regard to psychophysiological responses to stress.

\section{Time points}

Some consideration should be given to when outcomes are measured. Almost all the studies included at least one immediate post stress measurement, but only half of the studies included an additional measurement (mostly 30 or 60 minutes after cessation of the stressor), with only two studies including more than two follow-up measurements. Cytokine responses may be delayed for minutes or hours relative to ANS and HPA responses, because cytokines are produced by activated lymphocytes and macrophages [97-99]. This makes it difficult to detect the peak immune response when collecting only one or two samples, for example for the increase in IL-6 and IL-1 receptor agonists following psychosocial stress [85].

Additionally, in accordance with the allostatic load concept [100] the ability and time taken by the activated stress system to return to baseline (recovery period) might be an important factor in the link between stress and disease [99,101-103]. Moreover, repeated mental stress has been linked to a lack of habituation of the cortisol response [89,104] and plasma interleukin-6 [99] in subgroups of individuals. Thus future studies should have more frequent evaluation time points and investigate individual differences in the return-to-baseline values and in habituation patterns after repeated stress.

\section{Conclusions}

In summary, this review shows that there is limited evidence that autonomic and neuroendocrine function is altered after physical or psychological stress in patients with inflammatory rheumatic diseases compared with healthy subjects. In contrast, there is evidence that immune function is altered by stress in a manner specific to different rheumatic diseases, and thus real-life stressors could contribute to the maintenance or exacerbation of rheumatic diseases.

Future studies of stress, and particularly psychosocial stress, should have a follow-up with multiple measurement points, assess different physiological stress systems, and take into account stress appraisal. As individual differences in stress appraisal and stress response patterns might be important prognostic factors for disease progression, therapies that focus on stress management may be important adjuncts to traditional pharmacotherapy in the treatment of inflammatory rheumatic diseases. Stress induction studies could prove to be helpful for evaluating the effectiveness of these interventions $[105,106]$.

\section{Abbreviations}

ACTH: adrenocorticotropin hormone; ANS: autonomic nervous system; CNS central nervous system; $\mathrm{CRH}$ : corticotropin-releasing hormone; CRP: C-reactive protein; DHEA(-S): dehydroepiandosterone (sulfate); DMARDs: disease-modifying antirheumatic drugs; GH: growth hormone; HPA axis: hypothalamus-pituitary-adrenal axis; IFN- $\gamma$ : interferon- $\gamma$; IGF-I: insulin-like growth factor I; IL: interleukin; sIL-2 receptor: soluble interleukin-2 receptor; JIA: juvenile idiopathic arthritis; NK: natural killer cell; NPY: neuropeptide Y; NSAIDs: nonsteroidal anti-inflammatory drugs; PEP: pre-ejection period; RA; rheumatoid arthritis; SLE: systemic lupus erythematosus; TNF-a: tumor necrosis factor a.

\section{Competing interests}

The authors declare that they have no competing interests.

\section{Authors' contributions}

$\mathrm{SdB}$ and $\mathrm{AE}$ drafted the manuscript. The other authors critically revised it and approved the final content of the manuscript.

\section{Acknowledgements}

The study was supported by grants from the Dutch Arthritis Association ("Reumafonds").

\section{Author Details}

1Department of Medical Psychology, Radboud University Nijmegen Medical Centre, P.O. Box 9101, 6500 HB Nijmegen, The Netherlands, 2Department of Chemical Endocrinology, Radboud University Nijmegen Medical Centre, P.O. Box 9101, 6500 HB Nijmegen, The Netherlands and ${ }^{3}$ Department of Rheumatology, Radboud University Nijmegen Medical Centre, P.O. Box 9101, 6500 HB, Nijmegen, The Netherlands

Received: 8 September 2009 Revised: 14 January 2010 Accepted: 17 May 2010 Published: 17 May 2010

\section{References}

1. Cutolo M, Straub RH: Stress as a risk factor in the pathogenesis of rheumatoid arthritis. Neuroimmunomodulation 2006, 13:277-282.

2. Geenen R, Van Middendorp H, Bijlsma JW: The impact of stressors on health status and hypothalamic-pituitary-adrenal axis and autonomic nervous system responsiveness in rheumatoid arthritis. Ann N Y Acad Sci 2006, 1069:77-97.

3. Straub RH, Dhabhar FS, Bijlsma JW, Cutolo M: How psychological stress via hormones and nerve fibers may exacerbate rheumatoid arthritis. Arthritis Rheum 2005, 52:16-26.

4. Walker JG, Littlejohn GO, McMurray NE, Cutolo M: Stress system response and rheumatoid arthritis: a multilevel approach. Rheumatology (Oxford) 1999, 38:1050-1057.

5. Zautra AJ, Hamilton NA, Potter P, Smith B: Field research on the relationship between stress and disease activity in rheumatoid arthritis. Ann N Y Acad Sci 1999, 876:397-412

6. Miller GE, Chen E, Zhou ES: If it goes up, must it come down? Chronic stress and the hypothalamic-pituitary-adrenocortical axis in humans. Psychol Bull 2007, 133:25-45.

7. Ader R, Cohen N, Felten D: Psychoneuroimmunology: interactions between the nervous system and the immune system. Lancet 1995, 345:99-103

8. Anisman H, Merali Z: Cytokines, stress and depressive illness: brainimmune interactions. Ann Med 2003, 35:2-11.

9. Eskandari F, Webster Jl, Sternberg EM: Neural immune pathways and their connection to inflammatory diseases. Arthritis Res Ther 2003 , 5:251-265.

10. Straub RH, Cutolo M: Involvement of the hypothalamic--pituitaryadrenal/gonadal axis and the peripheral nervous system in 
rheumatoid arthritis: viewpoint based on a systemic pathogenetic role. Arthritis Rheum 2001, 44:493-507.

11. Calcagni E, Elenkov I: Stress system activity, innate and Thelper cytokines, and susceptibility to immune-related diseases. Ann N Y Acad Sci 2006, 1069:62-76.

12. Harbuz M: Neuroendocrine function and chronic inflammatory stress. Exp Physiol 2002, 87:519-525.

13. Kiecolt-Glaser JK, McGuire L, Robles TF, Glaser R: Psychoneuroimmunology and psychosomatic medicine: back to the future. Psychosom Med 2002, 64:15-28.

14. Kiecolt-Glaser JK, McGuire L, Robles TF, Glaser R: Psychoneuroimmunology: psychological influences on immune function and health. J Consult Clin Psychol 2002, 70:537-547.

15. Segerstrom SC, Miller GE: Psychological stress and the human immune system: a meta-analytic study of 30 years of inquiry. Psychol Bull 2004 130:601-630.

16. Steptoe A, Hamer M, Chida Y: The effects of acute psychological stress on circulating inflammatory factors in humans: a review and metaanalysis. Brain Behav Immun 2007, 21:901-912.

17. Steptoe A: Psychophysiological Bases of Disease. In Comprehensive Clinical Psychology. Health Psychology Volume 8. Edited by: Johnston DW, Johnston M. Oxford: Pergamon Press; 1998:39-78.

18. Elenkov IJ, Wilder RL, Chrousos GP, Vizi ES: The sympathetic nerve--an integrative interface between two supersystems: the brain and the immune system. Pharmacol Rev 2000, 52:595-638.

19. Maier SF, Watkins LR: Cytokines for psychologists: implications of bidirectional immune-to-brain communication for understanding behavior, mood, and cognition. Psychol Rev 1998, 105:83-107.

20. McEwen BS: Protective and damaging effects of stress mediators. $N$ Engl J Med 1998, 338:171-179.

21. Chrousos GP: The hypothalamic-pituitary-adrenal axis and immunemediated inflammation. N Engl J Med 1995, 332:1351-1362.

22. Zautra AJ: Comment on "stress-vulnerability factors as long-term predictors of disease activity in early rheumatoid arthritis". J Psychosom Res 2003, 55:303-304.

23. Kamarck TW, Lovallo WR: Cardiovascular reactivity to psychological challenge: conceptual and measurement considerations. Psychosom Med 2003, 65:9-21.

24. Dickerson SS, Kemeny ME: Acute stressors and cortisol responses: a theoretical integration and synthesis of laboratory research. Psychol Bull 2004, 130:355-391.

25. Kunz-Ebrecht SR, Mohamed-Ali V, Feldman PJ, Kirschbaum C, Steptoe A: Cortisol responses to mild psychological stress are inversely associated with proinflammatory cytokines. Brain Behav Immun 2003, 17:373-383.

26. Straub RH, Baerwald CG, Wahle M, Janig W: Autonomic dysfunction in rheumatic diseases. Rheum Dis Clin North Am 2005, 31:61-75. viii

27. Harbuz MS, Jessop DS: Is there a defect in cortisol production in rheumatoid arthritis? Rheumatology (Oxford) 1999, 38:298-302.

28. Jessop DS, Harbuz MS: A defect in cortisol production in rheumatoid arthritis: why are we still looking? Rheumatology (Oxford) 2005, 44:1097-1100

29. Geenen R, Godaert GL, Jacobs JW, Peters ML, Bijlsma JW: Diminished autonomic nervous system responsiveness in rheumatoid arthritis of recent onset. J Rheumatol 1996, 23:258-264.

30. Geenen R, Godaert GL, Heijnen CJ, Vianen ME, Wenting MJ, Nederhoff MG, Bijlsma JW: Experimentally induced stress in rheumatoid arthritis of recent onset: effects on peripheral blood lymphocytes. Clin Exp Rheumatol 1998, 16:553-559.

31. Hogarth MB, Judd L, Mathias CJ, Ritchie J, Stephens D, Rees RG: Cardiovascular autonomic function in systemic lupus erythematosus. Lupus 2002, 11:308-312.

32. Perry F, Heller PH, Kamiya J, Levine JD: Altered autonomic function in patients with arthritis or with chronic myofascial pain. Pain 1989, 39:77-84

33. Shalimar, Handa R, Deepak KK, Bhatia M, Aggarwal P, Pandey RM: Autonomic dysfunction in systemic lupus erythematosus. Rheumatol Int 2006, 26:837-840.

34. Kurtais $Y$, Tur BS, Elhan AH, Erdogan MF, Yalcin P: Hypothalamic-pituitaryadrenal hormonal responses to exercise stress test in patients with rheumatoid arthritis compared to healthy controls. J Rheumatol 2006, 33:1530-1537.
35. Pool AJ, Whipp BJ, Skasick AJ, Alavi A, Bland JM, Axford JS: Serum cortisol reduction and abnormal prolactin and CD4+/CD8+ T-cell response as a result of controlled exercise in patients with rheumatoid arthritis and systemic lupus erythematosus despite unaltered muscle energetics. Rheumatology (Oxford) 2004, 43:43-48.

36. Veldhuijzen van Zanten JJ, Ring C, Carroll D, Kitas GD: Increased C reactive protein in response to acute stress in patients with rheumatoid arthritis. Ann Rheum Dis 2005, 64:1299-1304.

37. Veldhuijzen van Zanten JJ, Kitas GD, Carroll D, Ring C: Increase in systemic vascular resistance during acute mental stress in patients with rheumatoid arthritis with high-grade systemic inflammation. Biol Psychol 2008, 77:106-110.

38. Dekkers JC, Geenen R, Godaert GL, Glaudemans KA, Lafeber FP, van Doornen LJ, Bijlsma JW: Experimentally challenged reactivity of the hypothalamic pituitary adrenal axis in patients with recently diagnosed rheumatoid arthritis. J Rheumatol 2001, 28:1496-1504.

39. Edwards RR, Wasan AD, Bingham CO III, Bathon J, Haythornthwaite JA, Smith MT, Page GG: Enhanced reactivity to pain in patients with rheumatoid arthritis. Arthritis Res Ther 2009, 11:R61.

40. Jacobs R, Pawlak CR, Mikeska E, Meyer-Olson D, Martin M, Heijnen CJ, Schedlowski M, Schmidt RE: Systemic lupus erythematosus and rheumatoid arthritis patients differ from healthy controls in their cytokine pattern after stress exposure. Rheumatology (Oxford) 2001, 40:868-875.

41. Motivala SJ, Khanna D, FitzGerald J, Irwin MR: Stress activation of cellular markers of inflammation in rheumatoid arthritis: protective effects of tumor necrosis factor alpha antagonists. Arthritis Rheum 2008, 58:376-383

42. Palm S, Hinrichsen H, Barth J, Halabi A, Ferstl R, Tolk J, Kirsten R, Kirch W: Modulation of lymphocyte subsets due to psychological stress in patients with rheumatoid arthritis. Eur I Clin Invest 1992, 22(Suppl 1):26-29.

43. Hinrichsen H, Barth J, Ferstl R, Kirch W: Changes of immunoregulatory cells induced by acoustic stress in patients with systemic lupus erythematosus, sarcoidosis, and in healthy controls. Eur $J$ Clin Invest 1989, 19:372-377.

44. Hinrichsen H, Barth J, Ruckemann M, Ferstl R, Kirch W: Influence of prolonged neuropsychological testing on immunoregulatory cells and hormonal parameters in patients with systemic lupus erythematosus. Rheumatol Int 1992, 12:47-51.

45. Pawlak CR, Jacobs R, Mikeska E, Ochsmann S, Lombardi MS, Kavelaars A, Heijnen CJ, Schmidt RE, Schedlowski M: Patients with systemic lupus erythematosus differ from healthy controls in their immunological response to acute psychological stress. Brain Behav Immun 1999, 13:287-302.

46. Roupe van der Voort C, Heijnen CJ, Wulffraat N, Kuis W, Kavelaars A: Stress induces increases in IL-6 production by leucocytes of patients with the chronic inflammatory disease juvenile rheumatoid arthritis: a putative role for alpha(1)-adrenergic receptors. J Neuroimmunol 2000, 110:223-229.

47. Altomonte L, Mirone L, Zoli A, Magaro M: Autonomic nerve dysfunction in systemic lupus erythematosus: evidence for a mild involvement. Lupus 1997, 6:441-444.

48. Kuis W, de Jong-de Vos van Steenwijk C, Sinnema G, Kavelaars A, Prakken B, Helders PJ, Heijnen CJ: The autonomic nervous system and the immune system in juvenile rheumatoid arthritis. Brain Behav Immun 1996, 10:387-398.

49. Stojanovich L, Milovanovich B, de Luka SR, Popovich-Kuzmanovich D, Bisenich V, Djukanovich B, Randjelovich T, Krotin M: Cardiovascular autonomic dysfunction in systemic lupus, rheumatoid arthritis, primary Sjogren syndrome and other autoimmune diseases. Lupus 2007, 16:181-185.

50. Toussirot E, Bahjaoui-Bouhaddi M, Poncet JC, Cappelle S, Henriet MT, Wendling $D$, Regnard J: Abnormal autonomic cardiovascular control in ankylosing spondylitis. Ann Rheum Dis 1999, 58:481-487.

51. Leden I, Eriksson A, Lilja B, Sturfelt G, Sundkvist G: Autonomic nerve function in rheumatoid arthritis of varying severity. Scand J Rheumatol 1983, 12:166-170.

52. Piha SJ, Voipio-Pulkki LM: Elevated resting heart rate in rheumatoid arthritis: possible role of physical deconditioning. Br J Rheumatol 1993, 32:212-215 
53. Cacioppo JT, Berntson GG, Malarkey WB, Kiecolt-Glaser JK, Sheridan JF, Poehlmann KM, Burleson MH, Ernst JM, Hawkley LC, Glaser R: Autonomic, neuroendocrine, and immune responses to psychological stress: the reactivity hypothesis. Ann N Y Acad Sci 1998, 840:664-673.

54. Cai FZ, Lester S, Lu T, Keen H, Boundy K, Proudman SM, Tonkin A, Rischmueller M: Mild autonomic dysfunction in primary Sjogren's syndrome: a controlled study. Arthritis Res Ther 2008, 10:R31.

55. Ewing DJ, Clarke BF: Diagnosis and management of diabetic autonomic neuropathy. BrMed J (Clin Res Ed) 1982, 285:916-918.

56. Louthrenoo W, Ruttanaumpawan P, Aramrattana A, Sukitawut W: Cardiovascular autonomic nervous system dysfunction in patients with rheumatoid arthritis and systemic lupus erythematosus. QJM 1999, 92:97-102.

57. Kirschbaum C, Pirke KM, Hellhammer DH: The 'Trier Social Stress Test'--a tool for investigating psychobiological stress responses in a laboratory setting. Neuropsychobiology 1993, 28:76-81.

58. Buske-Kirschbaum A, Geiben A, Hollig H, Morschhauser E, Hellhammer D: Altered responsiveness of the hypothalamus-pituitary-adrenal axis and the sympathetic adrenomedullary system to stress in patients with atopic dermatitis. J Clin Endocrinol Metab 2002, 87:4245-4251.

59. Schommer NC, Hellhammer DH, Kirschbaum C: Dissociation between reactivity of the hypothalamus-pituitary-adrenal axis and the sympathetic-adrenal-medullary system to repeated psychosocial stress. Psychosom Med 2003, 65:450-460.

60. Kirschbaum C, Kudielka BM, Gaab J, Schommer NC, Hellhammer DH: Impact of gender, menstrual cycle phase, and oral contraceptives on the activity of the hypothalamus-pituitary-adrenal axis. Psychosom Med 1999, 61:154-162.

61. Kudielka BM, Schommer NC, Hellhammer DH, Kirschbaum C: Acute HPA axis responses, heart rate, and mood changes to psychosocial stress (TSST) in humans at different times of day. Psychoneuroendocrinology 2004, 29:983-992.

62. Kudielka BM, Buske-Kirschbaum A, Hellhammer DH, Kirschbaum C: HPA axis responses to laboratory psychosocial stress in healthy elderly adults, younger adults, and children: impact of age and gender. Psychoneuroendocrinology 2004, 29:83-98.

63. Hirano D, Nagashima M, Ogawa R, Yoshino S: Serum levels of interleukin 6 and stress related substances indicate mental stress condition in patients with rheumatoid arthritis. J Rheumato/ 2001, 28:490-495.

64. Tanno M, Nakajima A, Ishiwata T, Naito Z, Yoshino S: Effect of general anesthesia on the abnormal immune response in patients with rheumatoid arthritis. Clin Exp Rheumatol 2004, 22:727-732.

65. Straub RH, Pongratz G, Hirvonen H, Pohjolainen T, Mikkelsson M, LeirisaloRepo M: Acute cold stress in rheumatoid arthritis inadequately activates stress responses and induces an increase of interleukin 6. Ann Rheum Dis 2009, 68:572-578.

66. Baerwald CG, Laufenberg $M$, Specht $T$, von Wichert $P$, Burmester GR, Krause A: Impaired sympathetic influence on the immune response in patients with rheumatoid arthritis due to lymphocyte subset-specific modulation of beta 2-adrenergic receptors. Br J Rheumatol 1997, 36:1262-1269.

67. Miller LE, Justen HP, Scholmerich J, Straub RH: The loss of sympathetic nerve fibers in the synovial tissue of patients with rheumatoid arthritis is accompanied by increased norepinephrine release from synovial macrophages. FASEB J 2000, 14:2097-2107.

68. Ishii H, Nagashima M, Tanno M, Nakajima A, Yoshino S: Does being easily moved to tears as a response to psychological stress reflect response to treatment and the general prognosis in patients with rheumatoid arthritis? Clin Exp Rheumatol 2003, 21:611-616.

69. Schmid-Ott G, Jacobs R, Jager B, Klages S, Wolf J, Werfel T, Kapp A, Schurmeyer T, Lamprecht F, Schmidt RE, Schedlowski M: Stress-induced endocrine and immunological changes in psoriasis patients and healthy controls. A preliminary study. Psychother Psychosom 1998, 67:37-42

70. Kraemer WJ, Ratamess NA: Hormonal responses and adaptations to resistance exercise and training. Sports Med 2005, 35:339-361.

71. Pedersen BK, Hoffman-Goetz L: Exercise and the immune system: regulation, integration, and adaptation. Physiol Rev 2000, 80:1055-1081.

72. Backonja MM, Walk D, Edwards RR, Sehgal N, Moeller-Bertram T, Wasan A, Irving G, Argoff C, Wallace M: Quantitative sensory testing in measurement of neuropathic pain phenomena and other sensory abnormalities. Clin J Pain 2009, 25:641-647.
73. Edwards RR, Sarlani E, Wesselmann U, Fillingim RB: Quantitative assessment of experimental pain perception: multiple domains of clinical relevance. Pain 2005, 114:315-319.

74. Edwards RR, Kronfli T, Haythornthwaite JA, Smith MT, McGuire L, Page GG: Association of catastrophizing with interleukin- 6 responses to acute pain. Pain 2008, 140:135-144.

75. Zautra AJ, Okun MA, Robinson SE, Lee D, Roth SH, Emmanual J: Life stress and lymphocyte alterations among patients with rheumatoid arthritis. Health Psychol 1989, 8:1-14.

76. Heim C, Ehlert U, Hellhammer DH: The potential role of hypocortisolism in the pathophysiology of stress-related bodily disorders. Psychoneuroendocrinology 2000, 25:1-35.

77. Harrington L, Affleck G, Urrows S, Tennen H, Higgins P, Zautra A, Hoffman $S:$ Temporal covariation of soluble interleukin-2 receptor levels, daily stress, and disease activity in rheumatoid arthritis. Arthritis Rheum 1993, 36:199-203.

78. Zautra AJ, Burleson MH, Matt KS, Roth S, Burrows L: Interpersonal stress, depression, and disease activity in rheumatoid arthritis and osteoarthritis patients. Health Psychol 1994, 13:139-148.

79. Zautra AJ, Hoffman JM, Matt KS, Yocum D, Potter PT, Castro WL, Roth S: An examination of individual differences in the relationship between interpersonal stress and disease activity among women with rheumatoid arthritis. Arthritis Care Res 1998, 11:271-279.

80. Davis MC, Zautra AJ, Younger J, Motivala SJ, Attrep J, Irwin MR: Chronic stress and regulation of cellular markers of inflammation in rheumatoid arthritis: implications for fatigue. Brain Behav Immun 2008, 22:24-32.

81. Kavelaars A, de Jong-de Vos van Steenwijk T, Kuis W, Heijnen CJ: The reactivity of the cardiovascular system and immunomodulation by catecholamines in juvenile chronic arthritis. Ann N Y Acad Sci 1998, 840:698-704.

82. Eggert M, Kluter A, Rusch D, Schmidt KL, Dotzlaw H, Schulz M, Pabst W, Boke J, Renkawitz R, Neeck G: Expression analysis of the glucocorticoid receptor and the nuclear factor-kB subunit p50 in lymphocytes from patients with rheumatoid arthritis. J Rheumatol 2002, 29:2500-2506.

83. Neeck G, Kluter A, Dotzlaw H, Eggert M: Involvement of the glucocorticoid receptor in the pathogenesis of rheumatoid arthritis. Ann N Y Acad Sci 2002, 966:491-495.

84. Schlaghecke R, Kornely E, Wollenhaupt J, Specker C: Glucocorticoid receptors in rheumatoid arthritis. Arthritis Rheum 1992, 35:740-744.

85. Steptoe A, Willemsen G, Owen N, Flower L, Mohamed-Ali V: Acute mental stress elicits delayed increases in circulating inflammatory cytokine levels. Clin Sci (Lond) 2001, 101:185-192.

86. Bohnen N, Nicolson N, Sulon J, Jolles J: Coping style, trait anxiety and cortisol reactivity during mental stress. J Psychosom Res 1991, 35:141-147

87. Hubert W, de Jong-Meyer R: Saliva cortisol responses to unpleasant film stimuli differ between high and low trait anxious subjects. Neuropsychobiology 1992, 25:115-120.

88. Schlotz W, Schulz P, Hellhammer J, Stone AA, Hellhammer DH: Trait anxiety moderates the impact of performance pressure on salivary cortisol in everyday life. Psychoneuroendocrinology 2006, 31:459-472.

89. Kirschbaum C, Prussner JC, Stone AA, Federenko I, Gaab J, Lintz D, Schommer N, Hellhammer DH: Persistent high cortisol responses to repeated psychological stress in a subpopulation of healthy men. Psychosom Med 1995, 57:468-474.

90. Pruessner JC, Gaab J, Hellhammer DH, Lintz D, Schommer N, Kirschbaum $\mathrm{C}$ : Increasing correlations between personality traits and cortisol stress responses obtained by data aggregation. Psychoneuroendocrinology 1997, 22:615-625.

91. Wirtz PH, Elsenbruch S, Emini L, Rudisuli K, Groessbauer S, Ehlert U: Perfectionism and the cortisol response to psychosocial stress in men. Psychosom Med 2007, 69:249-255.

92. Habra ME, Linden W, Anderson JC, Weinberg J: Type D personality is related to cardiovascular and neuroendocrine reactivity to acute stress. J Psychosom Res 2003, 55:235-245.

93. Gaab J, Rohleder N, Nater UM, Ehlert U: Psychological determinants of the cortisol stress response: the role of anticipatory cognitive appraisal. Psychoneuroendocrinology 2005, 30:599-610.

94. Rohrmann S, Hennig J, Netter P: Changing psychobiological stress reactions by manipulating cognitive processes. Int J Psychophysiol 1999, 33:149-161. 
95. Wirtz PH, Ehlert U, Emini L, Rudisuli K, Groessbauer S, Gaab J, Elsenbruch S, von Känel R: Anticipatory cognitive stress appraisal and the acute procoagulant stress response in men. Psychosom Med 2006, 68:851-858.

96. Wirtz PH, von Känel R, Emini L, Suter T, Fontana A, Ehlert U: Variations in anticipatory cognitive stress appraisal and differential proinflammatory cytokine expression in response to acute stress. Brain Behav Immun 2007, 21:851-859.

97. Febbraio MA, Ott P, Nielsen HB, Steensberg A, Keller C, Krustrup P, Secher $\mathrm{NH}$, Pedersen BK: Hepatosplanchnic clearance of interleukin- 6 in humans during exercise. Am J Physiol Endocrinol Metab 2003, 285:E397-E402.

98. Febbraio MA, Pedersen BK: Muscle-derived interleukin-6: mechanisms for activation and possible biological roles. FASEB J 2002, 16:1335-1347.

99. von Känel R, Kudielka BM, Preckel D, Hanebuth D, Fischer JE: Delayed response and lack of habituation in plasma interleukin- 6 to acute mental stress in men. Brain Behav Immun 2006, 20:40-48.

100. McEwen BS: Allostasis and allostatic load: implications for neuropsychopharmacology. Neuropsychopharmacology 2000 22:108-124

101. Earle TL, Linden W, Weinberg J: Differential effects of harassment on cardiovascular and salivary cortisol stress reactivity and recovery in women and men. J Psychosom Res 1999, 46:125-141.

102. Gerin W, Pickering TG: Association between delayed recovery of blood pressure after acute mental stress and parental history of hypertension. J Hypertens 1995, 13:603-610.

103. Linden W, Earle TL, Gerin W, Christenfeld N: Physiological stress reactivity and recovery: conceptual siblings separated at birth? J Psychosom Res 1997, 42:117-135.

104. Kudielka BM, von Känel R, Preckel D, Zgraggen L, Mischler K, Fischer JE: Exhaustion is associated with reduced habituation of free cortisol responses to repeated acute psychosocial stress. Biol Psychol 2006, 72:147-153

105. Gaab J, Blattler N, Menzi T, Pabst B, Stoyer S, Ehlert U: Randomized controlled evaluation of the effects of cognitive-behavioral stress management on cortisol responses to acute stress in healthy subjects. Psychoneuroendocrinology 2003, 28:767-779.

106. Hammerfald K, Eberle C, Grau M, Kinsperger A, Zimmermann A, Ehlert U, Gaab J: Persistent effects of cognitive-behavioral stress management on cortisol responses to acute stress in healthy subjects--a randomized controlled trial. Psychoneuroendocrinology 2006, 31:333-339.

doi: 10.1186/ar3016

Cite this article as: de Brouwer et al., Experimental stress in inflammatory rheumatic diseases: a review of psychophysiological stress responses Arthritis Research \& Therapy 2010, 12:R89

\section{Submit your next manuscript to BioMed Central} and take full advantage of:

- Convenient online submission

- Thorough peer review

- No space constraints or color figure charges

- Immediate publication on acceptance

- Inclusion in PubMed, CAS, Scopus and Google Scholar

- Research which is freely available for redistribution

Submit your manuscript at www.biomedcentral.com/submit
C) Biomed Central 